\title{
Invariance of Angular Threshold Computation in a Wide-Field Looming-Sensitive Neuron
}

\author{
Fabrizio Gabbiani, ${ }^{1,2}$ Chunhui Mo, ${ }^{1}$ and Gilles Laurent ${ }^{1}$ \\ ${ }^{1}$ Computation and Neural Systems Program, Division of Biology, California Institute of Technology, Pasadena, California \\ 91125, and 2Division of Neuroscience, Baylor College of Medicine, Houston, Texas 77030
}

The lobula giant motion detector (LGMD) is a wide-field bilateral visual interneuron in North American locusts that acts as an angular threshold detector during the approach of a solid square along a trajectory perpendicular to the long axis of the animal (Gabbiani et al., 1999a). We investigated the dependence of this angular threshold computation on several stimulus parameters that alter the spatial and temporal activation patterns of inputs onto the dendritic tree of the LGMD, across three locust species. The same angular threshold computation was implemented by LGMD in all three species. The angular threshold computation was invariant to changes in target shape (from solid squares to solid discs) and to changes in target texture (checkerboard and concentric patterns). Finally, the angular threshold computation did not depend on object approach angle, over at least $135^{\circ}$ in the horizontal plane. A two-dimensional model of the responses of the LGMD based on linear summation of motion-related excitatory and size-dependent inhibitory inputs successfully reproduced the experimental results for squares and discs approaching perpendicular to the long axis of the animal. Linear summation, however, was unable to account for invariance to object texture or approach angle. These results indicate that LGMD is a reliable neuron with which to study the biophysical mechanisms underlying the generation of complex but invariant visual responses by dendritic integration. They also suggest that invariance arises in part from non-linear integration of excitatory inputs within the dendritic tree of the LGMD.

Key words: invariant responses; looming; locust; LGMD; DCMD; collision avoidance
Invariance of neuronal responses is a key aspect of sensory processing. It characterizes the extraction of specific features in a stimulus, independent of their context (Cavanagh, 1978). Some invariant responses, such as those underlying the detection of edges in an image regardless of contrast level, rely on gain control mechanisms that are relatively well understood at the cellular and network levels (Galarreta and Hestrin, 1998; Sanchez-Vives et al., $2000 \mathrm{a}, \mathrm{b})$. In the inferotemporal visual area (IT) of the monkey, evidence suggests that the responses of many neurons are invariant to translation and scale transformations (Schwartz et al., 1983; Rolls and Baylis, 1986), a possible prerequisite for object identification (Sary et al., 1993). Although little is known about how this type of invariance arises, some authors have recognized the need for mechanistic investigations and proposed models (Olshausen et al., 1993; Salinas and Abbott, 1997; Riesenhuber and Poggio, 1999). The neural basis of response invariance, however, remains poorly understood. It is not known whether it results from network properties, from dendritic processing within single cells, or from combinations of both. We study two monosynaptically connected neurons in the visual system of the locust and

\footnotetext{
Received April 10, 2000; revised Oct. 10, 2000; accepted Oct. 18, 2000.

This work was supported by the German American Academic Council, by a National Institute on Deafness and Other Communication Disorders (National Institutes of Health) grant to G.L., by a National Science Foundation grant to C.K., and by the Sloan Center for Theoretical Neuroscience at Caltech. Experiments and data analysis were carried out by F.G. and C.M.; F.G. and G.L. co-wrote the paper. We thank Dr. A. Hassanali (ICIPE) and Dr. H. Krapp (University of Bielefeld) for kindly furnishing L. migratoria and S. gregaria specimens, as well as Dr. D. Sheinberg for help in setting up the data acquisition system. F.G. and C.M. thank Dr. C. Koch for many discussions and his encouragement over the course of this project.

Correspondence should be addressed to Dr. F. Gabbiani, Division of Neuroscience, One Baylor Plaza, Houston, TX 77030 (E-mail: gabbiani@bcm.tmc.edu) or to Dr. G. Laurent, Division of Biology, Caltech, Pasadena, CA 91125 (E-mail: laurentg@caltech.edu).

Copyright (C) 2001 Society for Neuroscience 0270-6474/01/210314-16\$15.00/0
}

demonstrate that they exhibit invariance properties to many attributes of the stimuli that best excite them. Because the neuronal processing leading to these invariant responses is thought to occur within the dendritic tree of one of these neurons, it might prove appropriate to study the biophysical mechanisms underlying the generation of invariant visual responses in single neurons.

The lobula giant motion detector (LGMD) is located in the third visual neuropil of the locust optic lobe (Strausfeld and Nässel, 1981). Its dendritic tree consists of three subfields that arborize in the lobula (O'Shea and Williams, 1974). The largest subfield receives excitatory inputs from afferents that are sensitive to local motion over the whole visual hemifield, whereas the remaining two subfields are thought to receive feedforward inhibitory projections that are size dependent (Palka, 1967; Rowell et al., 1977). LGMD synapses onto the descending contralateral motion detector (DCMD) (Killmann et al., 1999), a neuron the large axon of which projects to thoracic motor centers responsible for the generation of jump and flight steering maneuvers (Pearson et al., 1980; Simmons, 1980; Robertson and Pearson, 1983). The synaptic connection between LGMD and DCMD is so strong as to cause a one-to-one correspondence between presynaptic and postsynaptic spiking activity under visual stimulation (O'Shea and Williams, 1974; Rind, 1984). LGMD and DCMD are vigorously excited by objects looming toward the animal (Schlotterer, 1977; Rind and Simmons, 1992). The peak firing rate during approach of such looming objects signals the moment when the object reaches a fixed angular threshold size (Gabbiani et al., 1999a). This stimulus variable has in turn been related to the generation of escape and collision avoidance behaviors (Robertson and Johnson, 1993; Hatsopoulos et al., 1995).

Invariance of the peak firing time of LGMD and DCMD to 
changes of body temperature and stimulus luminance/contrast have already been demonstrated (Gabbiani et al., 1999a). In the present work, we investigate the extent to which the peak firing time of LGMD/DCMD - and thus the angular threshold detection computation - changes as we vary parameters of the approaching object that are expected to directly affect the time course of the spatially distributed excitatory and inhibitory inputs impinging onto the dendrites of the LGMD.

\section{MATERIALS AND METHODS}

The experimental materials and methods used were similar to those of Gabbiani et al. (1999a). The following brief account mainly emphasizes the differences with that work.

Preparation. Experiments were performed on three different species of North American and African locusts. Male and female specimens of the species Schistocerca americana (American Bird Grasshopper) were taken from the laboratory colony 3-4 weeks after their final molt. Adult male specimens from the African species Schistocerca gregaria (Desert Locust) and Locusta migratoria (African Migratory Locust) were imported (USDA-APHIS permits 36933 and 42816, respectively) from the International Center for Insect Physiology and Ecology (ICIPE, Nairobi, Kenya) and the University of Bielefeld (Germany). After the animals were fixed to a plastic holder, the head was aligned under a microscope with reference points marked on a reticular grid inserted in one of the eyepieces. This procedure and the calibration protocol described in Gabbiani et al. (1999a) allowed us to reliably align the center of the locust eye with the center of the video monitor used for visual stimulation. Animals were prepared for electrophysiological recordings in one of the following three manners: (1) mounted dorsal side up on the plastic holder, frontal dissection of the head capsule, performed as in Gabbiani et al. (1999a); (2) mounted ventral side up, connectives exposed by an incision of the neck cuticle (Hatsopoulos et al., 1995); or (3) mounted dorsal side up, rectangular incision of the pronotum. The gut was grabbed with a pair of fine forceps, cut as close as possible to the mouth, and removed through an abdominal incision, thus exposing the connectives. In all cases, the preparation was bathed in locust saline (Laurent and Davidowitz, 1994).

Electrophysiology and data acquisition. Locusts were fixed to a clamp with their longitudinal body axis parallel to the stimulation screen, except for the turntable experiments described below. For preparation 1, the connective contralateral to the stimulated eye was placed in a suction electrode (Gabbiani et al., 1999a). Alternatively, in preparations 2 and 3, two hook electrodes made of $50 \mu \mathrm{m}$ (0.02 inch) stainless steel wire isolated up to the tip with H-Formvar (California Fine Wire Co., Grover City, CA) were placed around the connective. The two electrodes were electrically isolated from each other with Vaseline (Hatsopoulos et al., 1995). Extracellular signals were amplified with a differential AC amplifier (A-M Systems, Everett, WA) and a Brownlee amplifier (model 210A; Brownlee, San Jose, CA). They were acquired together with transistor transistor logic (TTL) pulses synchronizing the visual stimuli with the recordings (Gabbiani et al., 1999a) using a 12 bit A/D board (win30; United Electronic Industries, Watertown, MA) connected to a personal computer running the QNX real-time operating system (QSSL, Kanata, Ontario, Canada). Each recorded spike train was inspected visually; DCMD action potentials (typically the largest in the nerve cord) (Fig. $1 A$ ) were selected on-line using custom software written in C.

Stimulation. Stimuli were generated using a fast monochrome video monitor refreshed at a rate of $200 \mathrm{~Hz}$, as described in Gabbiani et al. (1999a). The sequences of video images simulated circular and square objects with various textures approaching on a collision course with the animal. Each image was computed by central projection from the center of the stimulated eye onto the screen plane (Gabbiani et al., 1999a, their Fig. 2A and Eq. 2). The distance eye-screen was $120 \mathrm{~mm}$. The parameters characterizing each object approach were the radius (half-size), $l$, of the approaching disk or square (in centimeters), and the simulated constant speed of approach, $v$ (in meters per second) (see Fig. $1 A$, inset). If we set $t=0$ as the time of expected collision and adopt the convention that $t<$ 0 before collision, then the velocity $v$ is also negative when the object is approaching (Gabbiani et al., 1999a, their Eq. 1). Because the objects approached at a constant speed, elementary trigonometry shows that the image sequence is characterized by the ratio of these two parameters $l /|v|$ (in milliseconds) (Gabbiani et al., 1999a, their Fig. 1A). The range of values used for $l /|v|$ was from 5 to $50 \mathrm{msec}$ (see species, shape, texture, and approach angle protocols below), corresponding to approach speeds between 2 and $10 \mathrm{~m} / \mathrm{sec}$ for half-sizes between 6 and $14 \mathrm{~cm}$ (Gabbiani et al., 1999a, their Table 1).

Species protocols. In the first series of experiments, we compared the responses across different locust species by presenting to each animal 10 repetitions of looming squares approaching at 5 values of $l /|v|$ pseudorandomly interleaved (from $l /|v|=10-50 \mathrm{msec}$ in steps of $10 \mathrm{msec} ; 50$ protocols total). An interval of $40 \mathrm{sec}$ was inserted between successive stimulus presentations to minimize habituation (Gabbiani et al., 1999a). The squares were black $\left(0 \mathrm{~cd} / \mathrm{m}^{2}\right)$ on a bright background $\left(95 \mathrm{~cd} / \mathrm{m}^{2}\right)$. This protocol was applied to $n=5$ specimens of the species $S$. gregaria, 3 L. migratoria, and 64 S. americana. An additional eight L. migratoria were stimulated 10 times with 10 different values of $l /|v|$ (from 5 to 50 msec in steps of $5 \mathrm{msec}$ ) interleaved pseudorandomly (100 protocols total; an experiment lasted $\sim 1.25 \mathrm{hr}$ ). This protocol was identical to the one used in Gabbiani et al. (1999a) and allowed for a more stringent test of the linear relationship between peak firing time and $l /|v|$, because an additional 50 trials at 5 supplementary $l /|v|$ values were gathered to test the linear model.

Shape protocol. In the second series of experiments we compared the responses to black disks and black squares looming toward the animal by presenting 10 times these two objects at 5 values of $l /|v|(l /|v|=10-50$ msec in steps of $10 \mathrm{msec}$; two shapes $\times 5 l /|v|$ values $\times 10$ repetitions $=$ 100 protocols; $n=5$ animals, $S$. americana). Both the shape of the presented object and the value of $l /|v|$ were interleaved pseudorandomly, with a 40 sec interstimulus interval.

Texture protocols. In the third series of experiments, we studied the effect of target texture by comparing the responses to a black looming square and a square textured with a $3 \times 3$ checkerboard pattern as illustrated in Figure $5 A$ (inset) $(l /|v|=10-50 \mathrm{msec}$ in steps of $10 \mathrm{msec} ; 100$ pseudorandomly interleaved protocols; $40 \mathrm{sec}$ interstimulus interval; $n=$ 12 animals, $5 \mathrm{~S}$. gregaria, $7 \mathrm{~S}$. americana). The background was bright with a luminance $I_{\mathrm{B}}=B \cdot I_{\max }\left(B=100 \%, I_{\max }=95 \mathrm{~cd} / \mathrm{m}^{2}\right)$, and the checkerboard pattern had five squares of luminance $I_{\mathrm{O}}=O \cdot I_{\max }$, and four squares of luminance $I_{\mathrm{P}}=P \cdot I_{\max }$ with $O=0 \%$ and $P=30 \%$. In two experiments, the contrast between the squares of the checkerboard pattern was increased by setting $P=70 \%$.

The fourth series of experiments was a modified version of the previous series, with a textured pattern consisting of four concentric squares as illustrated in Figure $6 A$ (inset). The luminance of the background, $I_{\mathrm{B}}$, and object, $I_{\mathrm{O}}, I_{\mathrm{P}}$, were identical to those of the previous series $(B=$ $100 \%, O=0 \%, P=30 \% ; 100$ pseudorandomly interleaved protocols; 40 sec interstimulus interval; $n=12$ animals, $9 \mathrm{~S}$. americana, $3 \mathrm{~L}$. migratoria).

Approach angle protocols. The last three series of experiments investigated the effect of target approach angle. Animals were fixed to a clamp attached to a turntable that allowed rotation of both the animal and the micromanipulators holding the recording electrodes around a virtual vertical axis passing through the center of the eye. The turntable was equipped with a graduated dial allowing angles to be read with an accuracy of $\pm 1^{\circ}$. We denote by $0^{\circ}$ the position for which the longitudinal body axis is perpendicular to the stimulation screen (i.e., the animal faces the screen; see Fig. $7 A$, inset). Positive angles denote counterclockwise rotation, and negative angles denote clockwise rotations. The turntable could be positioned from $135^{\circ}$ (the object approaches from the same side as the stimulated eye, almost from the back) to $-45^{\circ}$ (the object approaches from the side contralateral to the stimulated eye). These experiments were performed using only the third dissection described above (see Preparation).

In the fifth series of experiments, the effect of approaching angle was tested at two positions: $0^{\circ}$ and $90^{\circ}$. During the first six experiments, the angles were alternated between $0^{\circ}$ and $90^{\circ}$, and the value of $l /|v|(l /|v|=$ $10-50 \mathrm{msec}$ in steps of $10 \mathrm{msec}$ ) were varied pseudorandomly from trial to trial $(2$ positions $\times 5 l /|v|$ values $\times 10$ repetitions $=100$ protocols $)$. During the next seven experiments, the angle was fixed at $0^{\circ}$ for the first 50 trials (pseudorandomly interleaved values of $l /|v|$ ) and then at $90^{\circ}$ for the remaining 50 trials $(n=13$ animals total, $S$. americana; $40 \mathrm{sec}$ interstimulus interval).

In the sixth series of experiments, the effect of approaching angle was tested at four positions on the same side as the stimulated eye $\left(0^{\circ}, 45^{\circ}\right.$, $90^{\circ}$, and $135^{\circ}$, from front to back) and 3 values of $l /|v|(l /|v|=10,30$, and $50 \mathrm{msec}$ ). At the end of a trial the animal was moved from one position to the next, and a new value of $l /|v|$ was chosen pseudorandomly. Each combination of position and $l /|v|$ value was repeated 10 times (4 posi- 
A

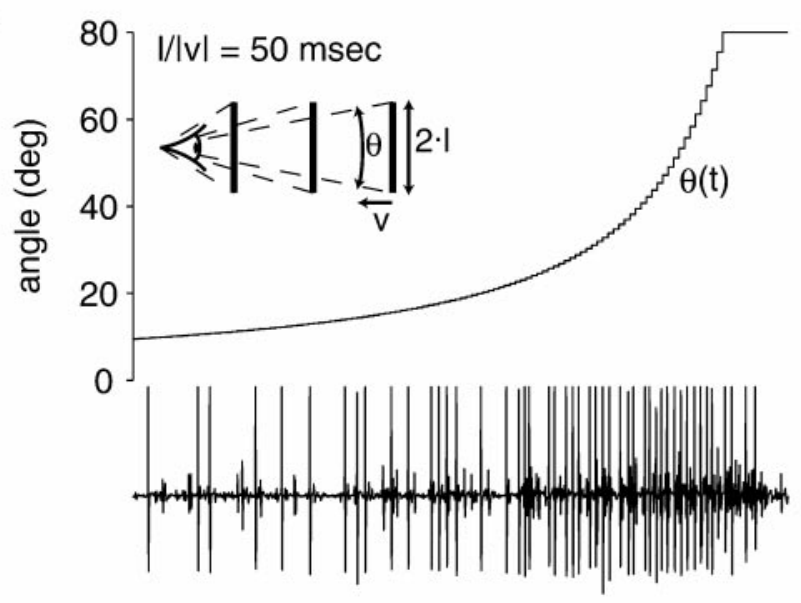

B

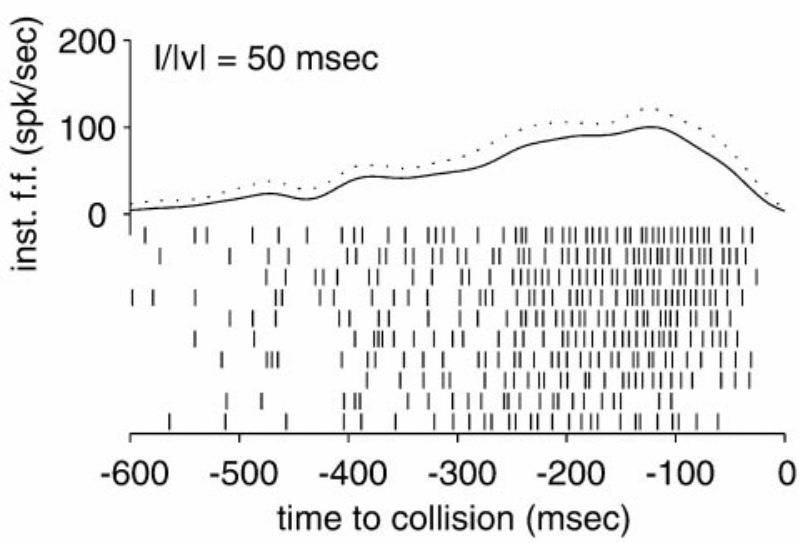

C
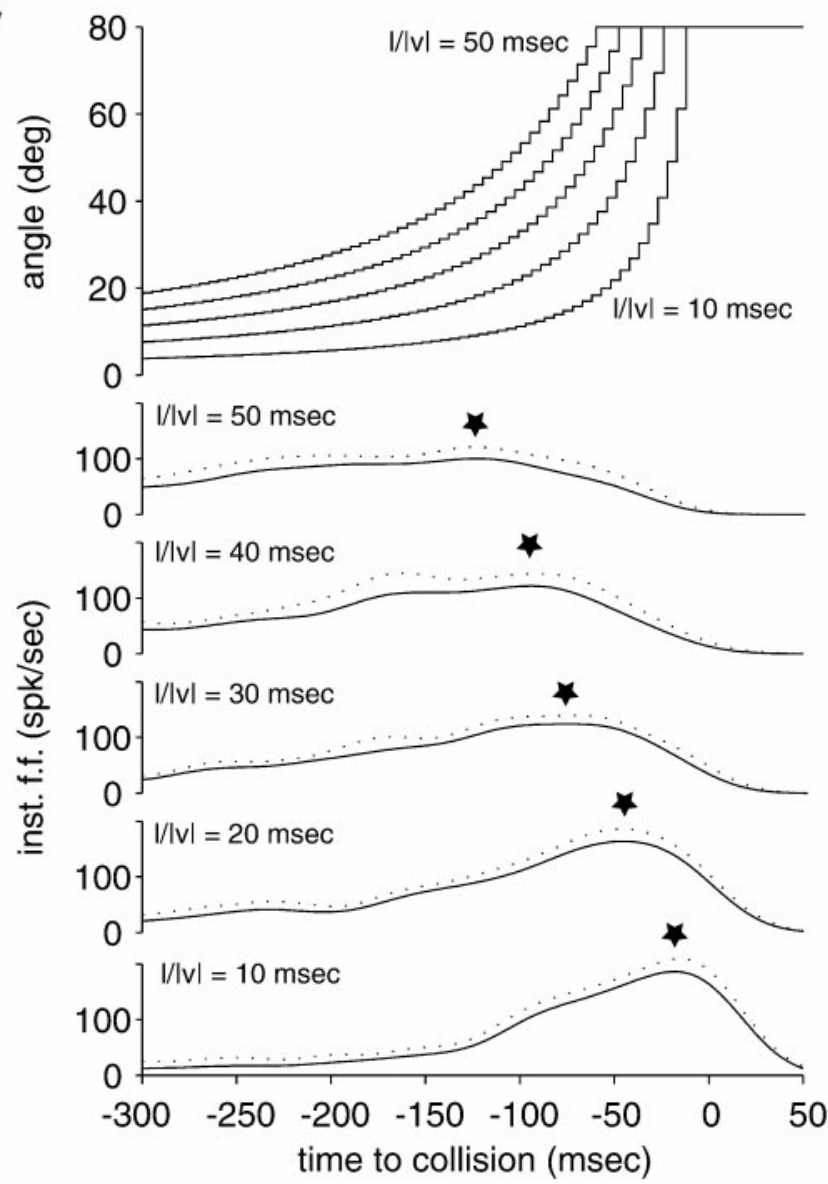

Figure 1. Responses of DCMD to simulated approaches of looming squares (L. migratoria). $A$, The time course of the angular size, $\theta(t)$, subtended by the object on the retina (inset) is illustrated on top $(l /|v|=50 \mathrm{msec}$ ). Each jump in angular size corresponds to a video screen refresh (see Materials and Methods). Bottom panel, Extracellular recording obtained from the connective contralateral to the stimulated eye. $B$, Ten repetitions of each stimulus were presented, and spike occurrence times were obtained by thresholding the recorded extracellular signals. The corresponding spike rasters are illustrated on the bottom (top raster corresponds to extracellular trace in $A$ ). $C$, The time of peak firing rate ( $\star$ ) shifted consistently toward collision as the stimulation parameter $l /|v|$ decreased. The mean peak firing times (obtained from similar graphs) and their SDs (obtained from the repetitions of each stimulus) are plotted as a function of $l /|v|$ in Figures 3-7.

tions $\times 3 l /|v|$ values $\times 10$ repetitions $=120$ protocols; $n=5$ animals, $S$. americana; $40 \mathrm{sec}$ interstimulus interval).

In the seventh series of experiments, five positions close to $0^{\circ}$ (animal facing the screen) were investigated $\left(-45^{\circ},-22.5^{\circ}, 0^{\circ}, 22.5^{\circ}\right.$, and $\left.45^{\circ}\right)$ at 3 values of $l /|v|(l /|v|=10,30$, and $50 \mathrm{msec})$. At the end of a trial, the animal was moved from one position to the next, and a new value of $l /|v|$ was chosen pseudorandomly. Each combination of position and $l /|v|$ value was repeated eight times $(5$ positions $\times 3 l /|v|$ values $\times 8$ repetitions $=$ 120 protocols; $n=11$ animals, S. americana; $40 \mathrm{sec}$ interstimulus interval). In this experiment, recordings were obtained from both connectives by placing one hook electrode around each connective and amplifying the voltage signal of each channel with respect to a far reference electrode implanted in the body.

Data analysis. Data analysis methods followed closely those of Gabbiani et al. (1999a). The extracellular traces obtained in response to the various stimuli described above were thresholded to extract the spike occurrence times of the DCMD (Fig. $1 A$ ). Each spike train was smoothed with a $20 \mathrm{msec}$ Gaussian window to obtain an estimate of the instantaneous firing rate (Fig. 1B) (Gabbiani et al., 1999a). Estimates of the mean time of peak firing rate and its SD were obtained from the repetitions (from 8 to 10 ) of each trial (Fig. $1 B$ ). The timing of the peak consistently shifted closer to collision time as the parameter $l /|v|$ characterizing the stimulus was decreased (Fig. 1C). This dependence was studied by plotting the peak time as a function of $l /|v|$ (see Figs. 3-7). Linear regressions of peak firing time as a function of $l / v \mid$ were performed for each experiment separately (see Figs. 3-7) to obtain the slope, $\alpha$, and the intercept parameter, $\delta$, of the best linear fit together with their
SD (Press et al., 1992). The angular threshold angles (see Figs. 3-7) and their SD were computed from $\alpha$ using Equation 6 of Gabbiani et al. (1999a):

$$
\theta_{\text {thres }}=2 \cdot \tan ^{-1} \frac{1}{\alpha}
$$

and by error propagation, respectively (i.e., using the formula $\Delta f=$ $|d f / d x| \cdot \Delta x$, for $f=f(x)$ ) (Bevington and Robinson, 1992, sect. 3.2). The delay between the time when the angular threshold size is reached and the peak firing rate, $\delta$ (see Figs. 3-7), is equal to the intercept parameter obtained by the linear fit described above. Both mean threshold angles and mean delay values are illustrated in Figures 4-7 with their SDs. The SDs correspond to $68.3 \%$ confidence intervals on the mean values of these parameters, whereas two SDs correspond to 95.4\% confidence intervals (Press et al., 1992, sect. 15.6). All data analysis was performed using Matlab 5.2 (The MathWorks, Natick, MA).

Two-dimensional modeling of the responses of the LGMD. The time course of LGMD/DCMD firing rate in response to solid squares or discs approaching toward the center of the eye along a trajectory perpendicular to the body axis may be described by the following one-dimensional model:

$$
(g \circ \exp )(\log \psi(t-\delta)-\alpha \theta(t-\delta)) .
$$

In this equation, $(g \circ \exp )(\cdot)=g(\exp (\cdot))$ represents the composition of the exponential function and a static nonlinearity $g(\cdot)$ described in Gabbiani 

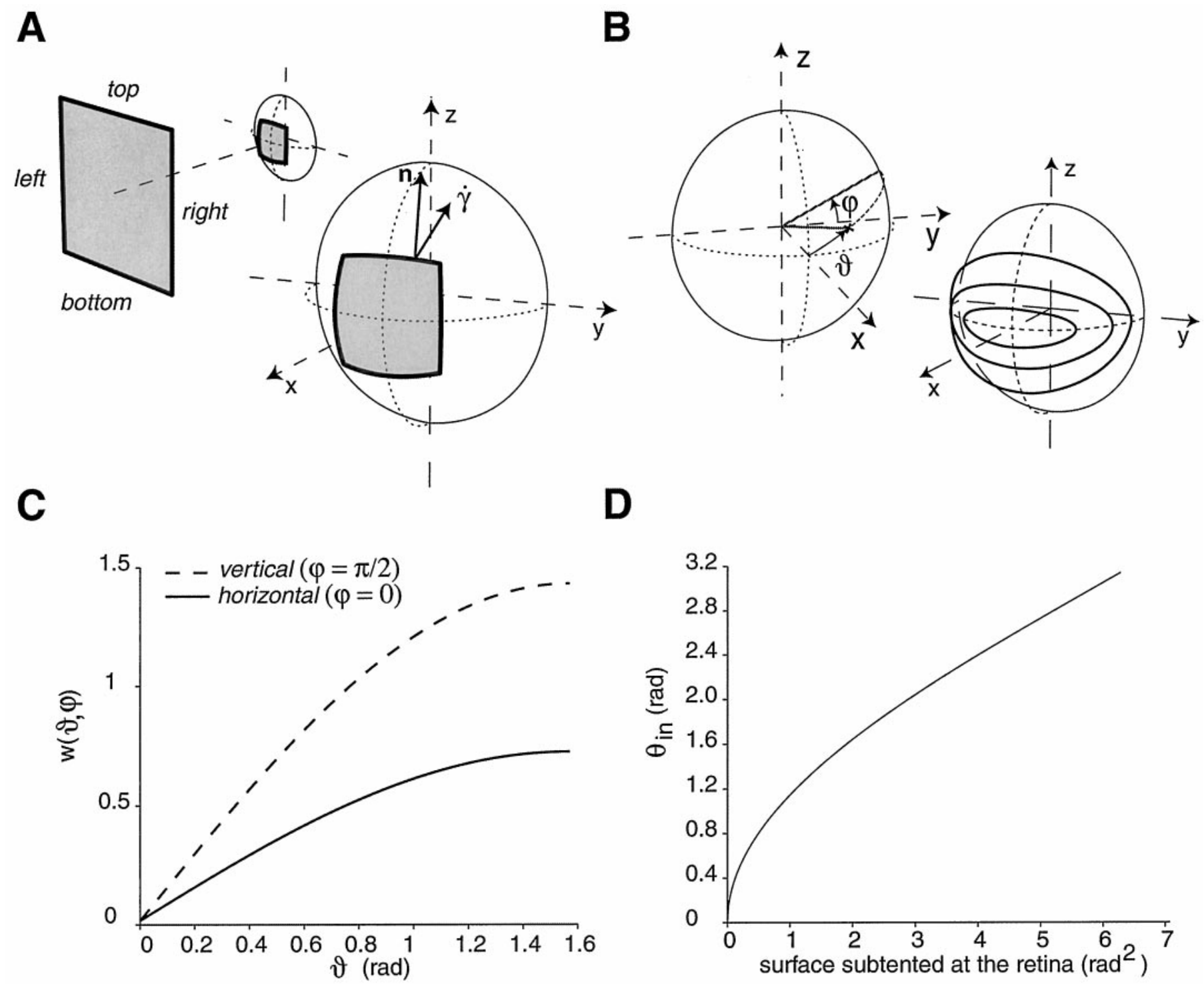

Figure 2. Two-dimensional modeling of LGMD/DCMD responses. $A$, The eye was described as a hemisphere; the image of a looming square on the eye was obtained by central projection (left diagram). The excitatory term of the response was calculated by projecting at each boundary point the instantaneous expansion vector $(\dot{\gamma})$ onto the unit normal $(\mathbf{n})$ to the boundary (optic flow vector; right diagram). The logarithm of the optic flow vector, multiplied by the inverse of a weight factor, was then integrated along the whole boundary (see Eq. 3). B, A point on the hemisphere (represented by a cross on the left diagram) was described by a pair of angles $(\vartheta, \varphi) . \varphi$ is the angle made with the $y$-axis by the projection of the point onto the $(y, z)$ plane. $\vartheta$ is the angle between the point and the $x$-axis. This angle is measured in the plane defined by the $x$-axis and the projection of the point onto the ( $y, z)$ plane. The right diagram illustrates ellipsoids of constant weight $a=2$ (Eq. 5). $C$, Value of the weight ( $a=2$ in Eq. 5) as a function of the angular distance to the center of the eye [i.e., the point with coordinates $(1,0,0)$ ] in the vertical, $(x, z)$-plane [dashed line, $\vartheta \in(0, \pi / 2), \varphi=\pi / 2]$ and the horizontal, $(x, y)$-plane [solid line, $\vartheta \in(0 ; \pi / 2), \varphi=0]$. $D$, Dependence of the inhibitory term $\theta_{\text {in }}$ on the surface subtended by the object on the retina (Eq. 6$)$.

et al. (1999a; their Eq. 11 and Discussion). The term $\log \psi(t-\delta)$, where $\psi(t)=1 / 2 \dot{\theta}(t)$ is the angular velocity of the edges of the objects on the retina, represents motion-dependent excitation, whereas $-\alpha \dot{\theta}(t-\delta)$ is an inhibitory term proportional to the angular size of the object. Equation 2 describes the approach of a solid square or circle based on the angle that it subtends with respect to the center of the eye and its temporal derivative. Hence, it cannot predict the responses to more complex (e.g., textured) stimuli or to the different approach angles explored in the present study. We therefore generalized Equation 2 to take explicitly into account the two-dimensional projection of the object on the retina. In the following, we will set the delay parameter $\delta=0$ for simplicity. This does not affect the generality of the arguments presented below because $\delta$ only causes a global translation of the firing rate along the time axis and thus a translation along the vertical axis of the linear relationship between the peak firing time relative to collision and $l /|v|$ (see Figs. 3-7). Similarly, the static nonlinearity $(g \circ \exp )(\cdot)$ does not influence the prediction of the peak firing rate time by the model because it is a monotonic function. It was therefore not taken further into consideration.

The eye was modeled as a hemisphere. Because the eye radius did not affect the output of the model, it was normalized to $R=1$ in the following description (see Appendix 1 for complete equations, including explicitly $R$ ). The object was projected onto the eye surface from the center of the hemisphere (Fig. 2A, left diagram). The motion-dependent excitatory input was obtained by integrating along each luminosity edge on the hemisphere the logarithm of the optic flow generated by the expanding object. Biophysically plausible mechanisms to compute this quantity have been described in insects (Hildreth and Koch, 1987; Reichardt et al., 1988; Zanker et al., 1999). Let $\left[p_{0} ; p_{1}\right]$ be a closed interval and $\gamma(t, p), p \in\left[p_{0} ; p_{1}\right]$ denote a parametrization of the edge. Each value of the parameter $p$ is required to describe the same physical point on the edge, independent of time. Let $\dot{\gamma}_{t_{0}}(p)=\partial /\left.\partial t \gamma(t, p)\right|_{t_{t=t_{0}}}$ denote the instantaneous velocity of expansion of the point $\gamma(t, p)$ at time 
$t=t_{0}$ and let $\mathbf{n}_{\mathbf{t}_{0}}(p)$ be the outward pointing normal unit vector (Fig. $2 A$, right diagram $)$. The excitation, $\operatorname{Ex}\left(t_{0}, l /|v|\right)$, caused by the motion of the edge on the retina was obtained by integrating the logarithm of the optic flow weighted by a factor $w\left(\gamma\left(t_{0}, p\right)\right)$ along the luminance edge:

$\operatorname{Ex}\left(t_{0}, \frac{l}{|v|}\right)=\frac{1}{2 \pi} \int_{\left[\mathrm{p} ; \mathrm{p}_{\mathrm{p}}\right]} d l_{\gamma}(p) \cdot w\left(\gamma\left(t_{0}, p\right)\right)^{-1} \cdot \log _{+}\left\langle\dot{\gamma}_{\mathrm{t}_{0}}(p) ; \mathbf{n}_{\mathrm{t}_{0}}(p)\right\rangle$.

In this equation $d l_{\gamma}(\cdot)$ denotes the line element associated with the curve $\gamma\left(t_{0}, \cdot\right)$ on the sphere (Dubrovin et al., 1991), and $\langle\cdot ; \cdot\rangle$ is the Euclidian scalar product. In Equation 3, the optic flow term has been renormalized by a constant $C_{\text {cutoff: }}$ :

$$
\log _{+}\left\langle\dot{\gamma}_{\mathrm{t}_{0}}(p) ; \mathbf{n}_{\mathrm{t}_{0}}(p)\right\rangle=\log \left\langle\dot{\gamma}_{\mathrm{t}_{0}}(p) ; \mathbf{n}_{\mathrm{t}_{0}}(p)\right\rangle-C_{\text {cutoff }} .
$$

The constant $C_{\text {cutoff }}$ was chosen so that $\log _{+}\left\langle\dot{\gamma}_{\mathrm{t}_{0}}(p) ; \mathbf{n}_{\mathrm{t}_{0}}(p)\right\rangle$ was equal to zero when the object subtended a half-angle $1 / 2 \theta_{\text {cutoff }}=1^{\circ}$ (corresponding to the optical resolution of the eye). During the remainder of the approach trajectory $\log _{+}\left\langle\dot{\gamma}_{\mathrm{t}_{0}}(p) ; \mathbf{n}_{\mathrm{t}_{0}}(p)\right\rangle$ was positive. The weight factor $w\left(\gamma\left(t_{0}, p\right)\right)$ depended on the distance of the local motion stimulus to the central axis of the eye. The weight factor mimicks the experimental decrease in LGMD sensitivity observed as a local motion stimulus is shifted from the eye center toward the periphery. Experimental evidence suggests that this sensitivity decreases faster in the vertical than in the horizontal plane. Iso-contour lines of equal weight were therefore chosen as elongated ellipsoids (Fig. $2 B$, right diagram). Let $(\vartheta \varphi)$ denote the coordinates of $\gamma\left(t_{0}, p\right)$ with respect to the coordinate system illustrated in Figure $2 B$, (left diagram). Then:

$$
w(\vartheta, \varphi)=|\sin \vartheta| \cdot \sqrt{\frac{\cos ^{2} \varphi}{a^{2}}+a^{2} \sin ^{2} \varphi}+\sin \frac{1}{2} \theta_{\text {cutoff }},
$$

with $a=2$ and $1 / 2 \theta_{\text {cutoff }}=1^{\circ}$. The choice $a=2$ yields the ellipsoidal contour lines illustrated in Figure $2 B$, and $1 / 2 \theta_{\text {cutoff }}=1^{\circ}$ causes the weight factor to saturate beyond the optical resolution of the eye. The dependence of $w(\vartheta, \varphi)$ on the angular distance to the eye center in the vertical and horizontal planes is illustrated in Figure $2 C$.

The size dependent inhibitory term $\operatorname{In}(t, l /|v|)$ was obtained by converting the surface, $S(t)$, covered by the object on the retina into an equivalent angle, $\theta_{\text {in }}(t)$ :

$$
\begin{aligned}
\operatorname{In}\left(t, \frac{l}{|v|}\right) & =\alpha \cdot \theta_{\text {in }}(t) \\
& =\alpha \cdot 2 \cos ^{-1}\left(1-\frac{S(t)}{2 \pi}\right) .
\end{aligned}
$$

Note that in this equation $S(t)$ is normalized by the surface of the eye $(=2 \pi=$ surface of a hemisphere of radius 1$)$. The value of $\theta_{\text {in }}$ as a function of $S$ is plotted in Figure 2D. For comparison with experimental data, the parameter $\alpha$ was set equal to 5 , a value that lies in the middle of the typical range observed for a large sample of LGMD neurons (Gabbiani et al., 1999a, their Fig. 6A).

When simulating approaches from angles other than $90^{\circ}$, the optic flow was integrated only across that portion of the object that effectively projected onto the eye. Similarly, only the surface of the object covering the retina was taken into account in Equation 6.

Appendix 1 gives a precise mathematical analysis of this model for an approaching circle. Appendix 2 describes the parametrization used for the edges of approaching squares, as well as the conditions describing the portion of the approaching square projecting onto the retina for approach angles different from $90^{\circ}$.

For the concentric square pattern, the excitatory term was obtained by linear summation of the excitatory terms corresponding to each of the concentric squares composing the pattern. If we denote by $\operatorname{Ex}_{\text {square }}(t, l /|v|)$ the excitation at time $t$ attributable to a square of half-size $l$ approaching at speed $|v|$, then:

$$
\operatorname{Ex}_{\text {concentric }}\left(t, \frac{l}{|v|}\right)=\sum_{\mathrm{k}=1}^{4} \operatorname{Ex}_{\text {square }}\left(t, \frac{l}{k \cdot|v|}\right)
$$

Similarly, the excitation term for the checkerboard pattern, $\mathrm{Ex}_{\text {checker }}{ }^{-}$ board $(t, l /|v|)$, was obtained by taking into account the two additional expanding vertical and horizontal inner edges. If the corresponding term is denoted by $\operatorname{Ex}_{\text {inner }}(t, l / 3 \cdot|v|)$, then:

$$
\operatorname{Ex}_{\text {checkerboard }}\left(t, \frac{l}{|v|}\right)=\operatorname{Ex}_{\text {square }}\left(t, \frac{l}{|v|}\right)+\operatorname{Ex}_{\text {inner }}\left(t, \frac{l}{3 \cdot|v|}\right) .
$$

Appendix 2 explains how $\operatorname{Ex}_{\text {inner }}(t, l / 3 \cdot|v|)$ is computed.

The symbolic algebra package Maple (Maple Inc., Waterloo, Ontario, Canada) was used to obtain numerical values for $\operatorname{Ex}(t, l /|v|)$ and $\operatorname{In}(t, l /|v|)$ under the tested experimental conditions. Although $\operatorname{In}(t, l /|v|)$ could be computed in closed form, the indefinite integral corresponding to Equation 3 could not be obtained in closed form for approaching squares. The integrand of Equation 3 was therefore computed symbolically and then integrated numerically using an 8th order Newton-Côtes quadrature method (Forsythe et al., 1977). Numerical calculation of $\operatorname{Ex}(t, l /|v|)$ and $\operatorname{In}(t, l /|v|)$ for all condition tested $(l /|v|=10,20,30,40,50 \mathrm{msec}$ for solid discs, squares, concentric square patterns, and checkerboard patterns approaching at $90^{\circ}$; same values of $l|| v \mid$ for solid squares approaching at angles from $90^{\circ}$ down to $-30^{\circ}$ in $15^{\circ}$ steps), and all combinations of model parameters tested $\left(a=1,2\right.$, and $1 / 2 \theta_{\text {cutoff }}=0^{\circ}, 1^{\circ}$ in Eq. 5) took approximately 1 week on a computer equipped with a $450 \mathrm{MHz}$ Pentium III processor (Intel, Santa Clara, CA). None of the results depended qualitatively on the choice of $a$ or $1 / 2 \theta_{\text {cutoff }}$ in Equation 5. Maple simulation files and Matlab functions reproducing Figures 8 and 9 may be obtained on the World Wide Web at http://glab.bcm.tmc.edu by following the "Publications" link and selecting the page corresponding to the title of this article.

\section{RESULTS}

The results described below are based on recordings and complete data analysis from 58 different animals. Part of the data used in Fig. $3 D$ was collected in 35 additional animals (S. americana) for different purposes.

\section{Is the angular threshold computation species dependent?}

The response of LGMD/DCMD in L. migratoria to the simulated approach of a solid square on a collision course with the animal is illustrated in Fig. $1 A$. The time course of visual stimulation on the retina is characterized by the ratio of the half-size of the square, $l$, and its approach velocity, $|v|$, (Fig. $1 A$, inset) [see also Materials and Methods and Gabbiani et al. (1999a)]. The response starts early during the approach, when the object reaches approximately $10^{\circ}$ in visual angle. It gradually increases and reaches its peak value before collision for large values of $l /|v|(\geq 10$ msec) (Fig. 1A-C). Each stimulation was repeated 8-10 times to obtain estimates of the mean time of peak firing rate and its SD (Fig. $1 B$ ). As may be seen in Figure $1 C$, the time of peak firing rate relative to collision $(\star)$ decreases systematically as the stimulation parameter $l /|v|$ decreases (i.e., as the approach velocity, $|v|$, of the moving object increases for a fixed object half-size, $l$ ). Figure $3 A$ replots the time of peak firing rate, $t_{\text {peak }}$, obtained from Figure $1 C$ as a function of the kinematic parameter $l /|v|$ characterizing the approach [see also Gabbiani et al. (1999a), their Figs. $3,4]$. To a very good approximation, $t_{\text {peak }}$ follows a linear relationship with $l /|v|$. Similar results have been reported for $S$. americana (Gabbiani et al., 1999a) and are illustrated in Figure 3B for one specimen of this species. Furthermore, as reported in Gabbiani et al. (1999a), this linear relationship between $t_{\text {peak }}$ and $l /|v|$ implies that the peak in firing rate occurs a fixed delay (5-40 msec on average in the present experiments) after the object has reached a fixed angular threshold size (between $15^{\circ}$ and $40^{\circ}$ on average) on the locust's retina, independent of the value of $l /|v|$ (Gabbiani et al., 1999a, their Fig. 5). To test further the linear dependence of peak firing time on $l /|v|$, an additional eight animals were presented looming stimuli at 10 values of $l /|v|$ with a protocol identical to the one used in Gabbiani et al. (1999a, their 
A

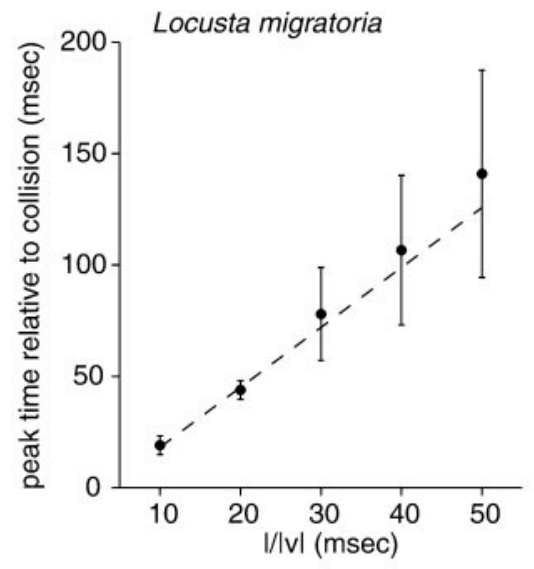

C

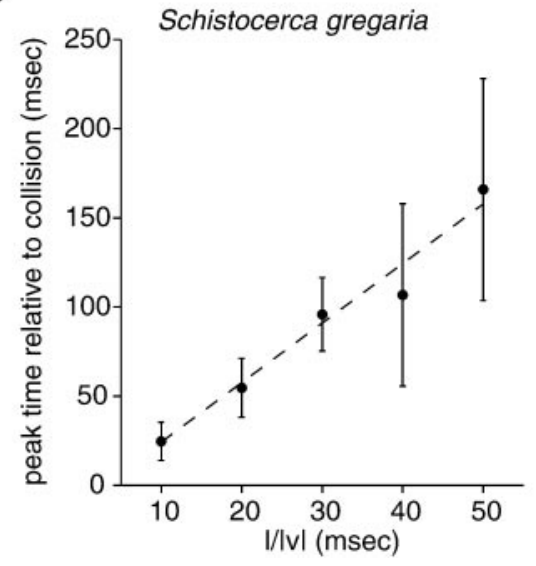

B

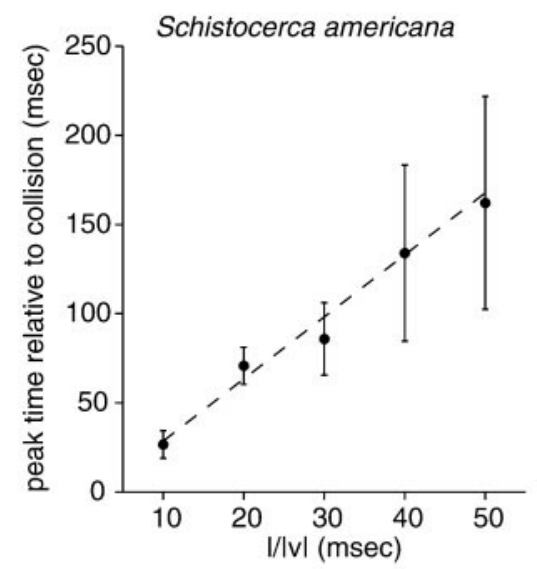

D

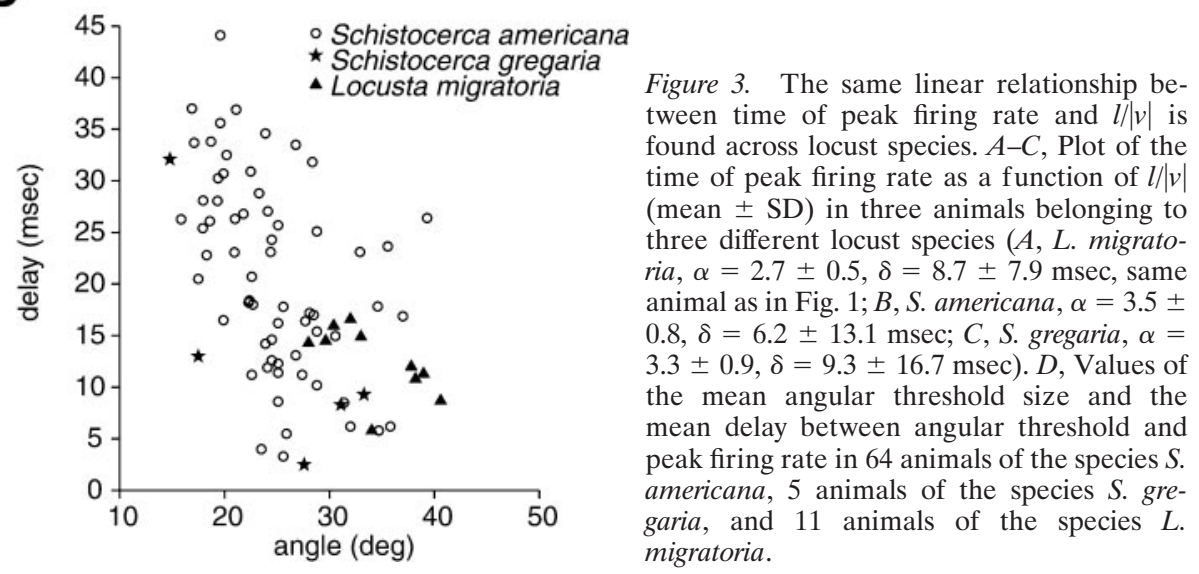

Fig. 4). The results of these experiments were also similar to those reported in $S$. americana. Thus, in L. migratoria as in S. americana, the peak firing rate can be seen as the output of an angular threshold detector and may contribute to trigger visually evoked escape behaviors [Hatsopoulos et al. (1995); see Gabbiani et al. (1999a) for a detailed discussion]. To ascertain whether this is also the case in a second African locust species, we performed similar experiments on $S$. gregaria. Figure $3 C$ illustrate the results of experiments performed on one animal of this species. In all experiments analyzed, the same linear relationship was found in both the American and African species. Figure $3 D$ plots the mean angular threshold sizes and delays observed for $S$. gregaria $(n=5$, $\star)$ and L. migratoria $(n=11,0)$. These values are well into the range observed in a large sample of animals from the American locust $S$. americana $(n=65, \bigcirc)$.

\section{Angular threshold computation and target shape}

Shape differences between solid objects looming toward the locust's eye translate into temporal and spatial differences in the stimulation of ommatidia on the retina. In the case of a disc and square having the same radius (or half-size), $l$, the temporal sequence of light intensities during approach is the same only along the two horizontal and vertical symmetry axes passing through the center of the objects. For a disc, the angular velocity of expansion on the retina at any given time points out from the center with a magnitude that is constant along the whole disc boundary. Because this vector is always perpendicular to the disc boundary, it coincides with the optic flow vector (i.e., the projec- tion of the expansion velocity vector onto the unit normal to the boundary) that can be measured locally at the retina (Appendix 1). By contrast, the angular velocity of expansion of a square is not always perpendicular to the square edges and its magnitude is greatest at its four corners (Fig. $4 A$, inset). After projection on the unit normal to the boundary, the optic flow vector magnitude is - on the contrary-greatest at the middle of an edge rather than at its corners. Both quantities may be computed from the equations given in Appendix 2. The ratio of the optic flow magnitude at the corner of a square to that of a disc decreases monotonically during approach from a peak value of 1 when both objects are far away to a minimum value of 0.5 at collision time. Thus, the motion stimulus generated by an expanding square is always less strong than the one generated by a disc, whereas the surface that a square of half-size $l$ subtends at the retina is always larger than the one subtended by a disc of radius $l$.

We investigated whether such shape differences affected the timing of peak firing by presenting to the same animal square (ם) and circular ( ) targets interleaved pseudo-randomly over the course of an experiment. Figure $4 A$ illustrates the outcome in one preparation: the differences in mean times of peak firing rate measured with both objects could not be distinguished from the intertrial variability (SD) (Fig. $4 A$, solid bars). Figure $4 B$ summarizes the outcome of five such experiments in different preparations by plotting mean angular thresholds versus mean delays obtained for square ( $\square$ ) and circular ( ) targets and their SDs (solid lines parallel to the axes). For each mean angle and delay 
Figure 4. The linear relationship between time of peak firing rate and $l /|v|$ is the same whether the target is a disc or a square. $A$, Plot of peak firing rate as a function of $l /|v|($ mean $\pm \mathrm{SD})$ for a square (target 1: $\mathbf{\square}, \alpha=5.1 \pm 0.8, \delta=11.8 \pm$ $12.8 \mathrm{msec}$ ) and a disc (target $2: \boldsymbol{\bullet}, \alpha=4.7 \pm 0.7$, $\delta=11.9 \pm 12.1 \mathrm{msec}$ ) measured in the same preparation. For clarity, only the largest SD is shown in one direction for each values of $l /|v|$. Bottom inset, The rate of angular expansion of a square target early during approach is largest at its corners where it equals 1.35 times the rate of expansion of a disc (for $l /|v|=50 \mathrm{msec}$ and $t=$ $-225 \mathrm{msec}$, approximate peak time). At this time, the optic flow vector at the corner (dotted line) is still almost identical, however, to the one of a disc. $B$, Plot of the delay versus the angular threshold (mean $\pm \mathrm{SD}$ ) for approaching squares and discs (illustrated by $\mathbf{\square}$ and $\boldsymbol{\bullet}$, respectively) measured in five different preparations (S. americana). Experiments performed on the same animal are connected by dashed lines. Solid lines illustrate SDs in one direction only for clarity. The extensive overlap of SDs for experiments performed on the same animal indicate no significant differences between the two stimulation conditions. $C$, Plot of the delay (mean $\pm \mathrm{SD}$ ) for target 2 versus target 1 for the same five preparations as in $B$. The dashed diagonal line represents identity. Delays cluster within $1 \mathrm{SD}$ of the diagonal, indicating no significant differences between the two conditions. $D$, Plot of the threshold angle (mean $\pm \mathrm{SD}$ ) for target 2 versus target 1 for the same five preparations as in $B$. In three of five experiments, threshold angles cluster within $1 \mathrm{SD}$ from the diagonal, indicating no significant differences between the two conditions. The remaining two cases were not statistically different at the $95.4 \%$ confidence level. Note that mean threshold angles for square targets are consistently smaller than those for discs (i.e., all points lie above the diagonal).
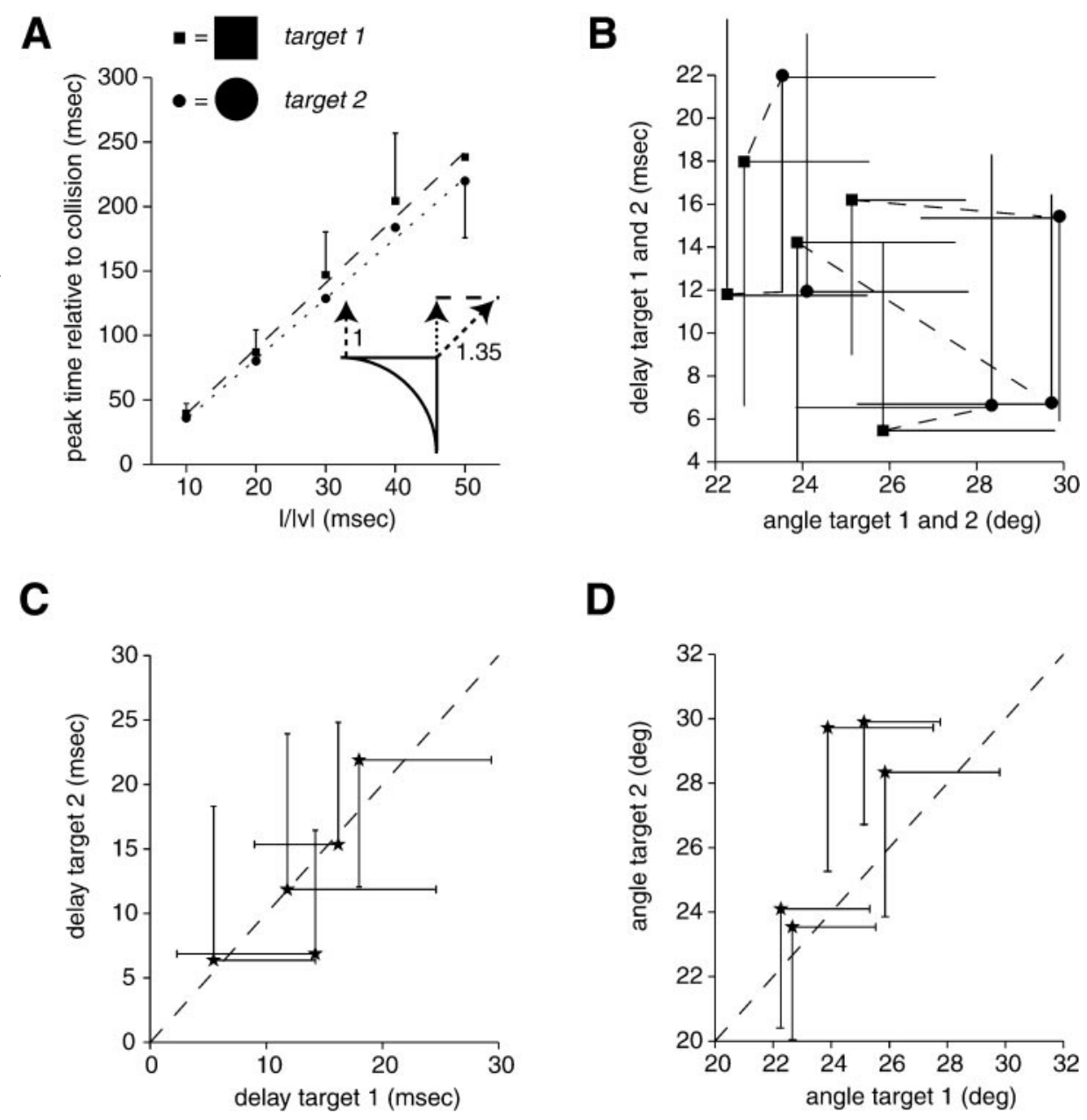

D

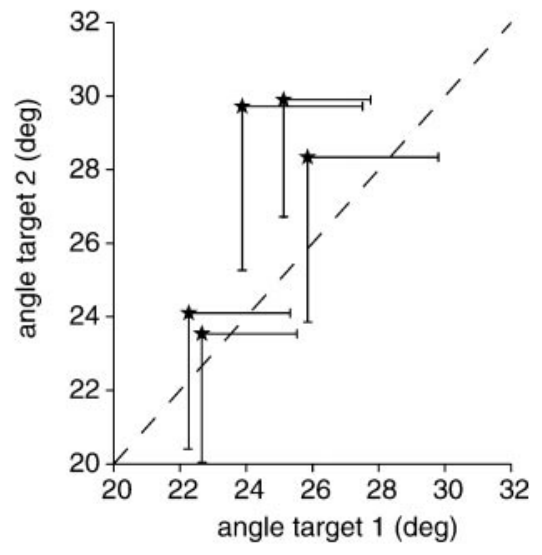

pair, the SDs outline the boundary of the two-dimensional $68.3 \%$ confidence region on their value (Gabbiani et al., 1999a, their Fig. 4B; Press et al., 1992, sect. 15.6). As can be seen from the plot, the two-dimensional confidence regions strongly overlap, and therefore mean angular thresholds and delays were not statistically different between the responses to these two stimulus conditions. Figure $4 C$ plots the mean delays for square versus circular targets and their SDs along the delay axis. No significant trend could be observed, and the data always lay within $1 \mathrm{SD}$ of the diagonal characterizing equal delays under both conditions. By contrast, the mean angular thresholds were consistently above the diagonal (Fig. 4D), corresponding to smaller average threshold angles, or equivalently earlier peak times for square targets. Differences in mean threshold angles were not significant in single preparations. In two cases, the SDs of the threshold angles did not intercept the diagonal, but these two points were located within two SDs of the diagonal and were thus not significantly different at the $95.4 \%$ level. The difference in mean threshold angles averaged over all preparations was small: $3.2 \pm 2.1^{\circ}$ (mean $\pm \mathrm{SD}$ ), but statistically significant (paired $t$ test, $p<0.005$ ). Thus, these results point to a small (i.e., not statistically significant in single preparations) but consistent trend across preparations of higher threshold angles and later peak firing times for disc targets.

\section{Angular threshold computation and target texture}

Variations in target texture will also result in different temporal and spatial activation of ommatidia on the locust's retina. We investigated whether such changes affected the time of peak firing rate during approach by comparing the responses obtained for solid square targets to two different textured targets: a checkerboard pattern (CBP) (Fig. $5 A$, inset) and a concentric squares pattern (CSP) (Fig. 6A, inset). For the CBP, the inner edges move in opposite directions as the object grows on the retina, whereas for the CSP, inner edges consistently follow the leading edges of the square although at a different instantaneous angular velocity. In both cases, the luminance of the pattern object was set at $30 \%$ (dark gray) and $0 \%$ (black) of the background luminance (95 $\left.\mathrm{cd} / \mathrm{m}^{2}\right)$; similar results were obtained in two animals with CBP relative luminances of $70 \%$ (light gray) and $0 \%$. Figures $5 A$ and $6 A$ illustrate the outcome of one experiment for the CBP (Fig. $5 A$, $\triangle$ ) and the CSP (Fig. $6 A, \triangle$ ), respectively. In both cases, the time of peak firing rate was statistically indistinguishable from the controls obtained with black square targets (Figs. $5 A, 6 A, \mathbf{\square}$ ). Figures $5 B$ and $6 B$ plot mean angular threshold versus delay obtained with the $\operatorname{CBP}(\triangle, n=10$ animals) and the $\operatorname{CSP}(\triangle, n=$ 12 animals), respectively, and compares them with those obtained in the same preparations with black square targets ( $\square$ ). Experiments performed on the same preparation are connected with a dashed line. In both cases, no consistent changes were observed with respect to the control condition $(\boldsymbol{\square})$. This was confirmed by first plotting the mean delays and SDs obtained in the control condition versus those of the two test conditions (CBP and CSP). Figures $5 C$ and $6 C$ illustrate the results for the CBP and CSP, respectively: the mean delays in the test versus control conditions 
A

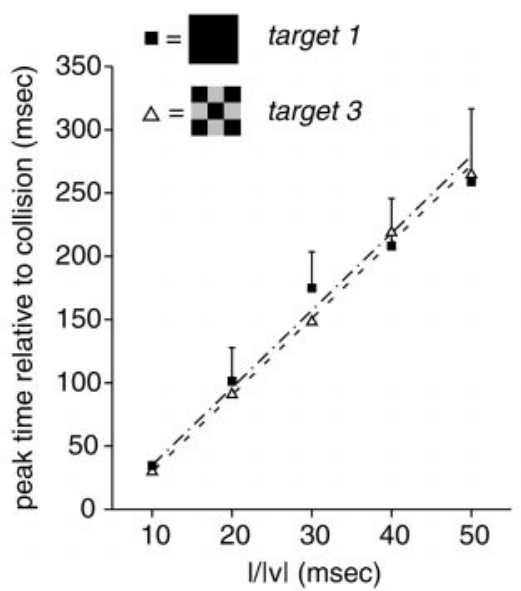

C

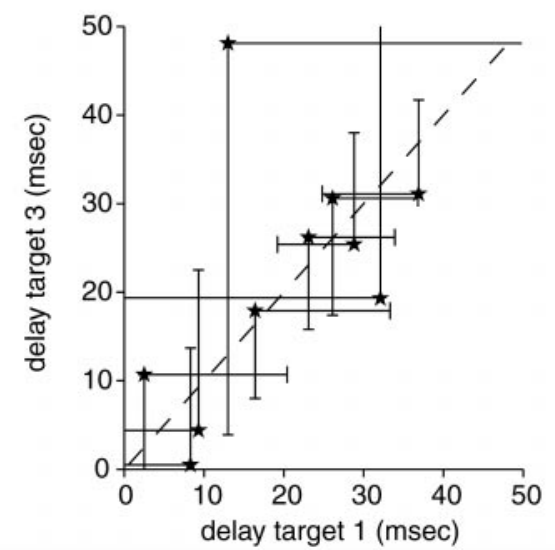

B

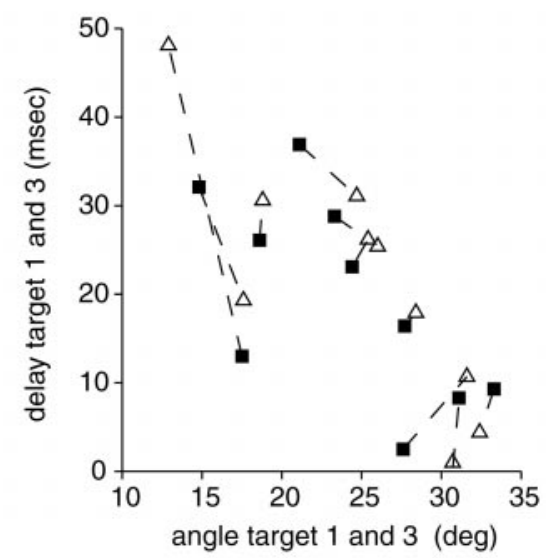

D

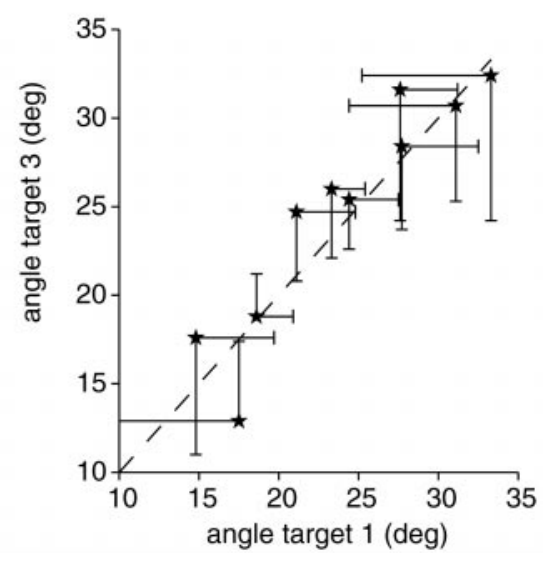

Figure 5. The linear relationship between time of peak firing rate and $l /|v|$ does not change with changes in target texture. $A$, Plot of peak firing rate as a function of $l /|v|$ (mean $\pm \mathrm{SD})$ for a square target (target 1: $\mathbf{\square}, \alpha=6.1 \pm 0.8, \delta=$ $26.1 \pm 10.7 \mathrm{msec})$ and a checkerboard textured target (target 3: $\triangle, \alpha=6.1 \pm 0.8, \delta=30.6 \pm$ $13.2 \mathrm{msec}$ ) measured in the same preparation (S. americana). For clarity only the largest SD is shown in one direction for each value of $l /|v|$. $B$, Plot of the mean delay for target 1 (ם) and target $3(\triangle)$ versus mean angular threshold in 10 different preparations $(5 \mathrm{~S}$. americana and 5 $S$. gregaria). Experiments performed on the same preparation are connected by a dashed line. $C$, Plot of the delay (mean $\pm \mathrm{SD}$ ) for target 3 versus the delay for target 1 in the same 10 preparations as in $B$. For clarity, SDs are illustrated in one direction only. The dashed diagonal line represents identity. Delays cluster within $1 \mathrm{SD}$ of the diagonal, indicating no significant differences between the two conditions. $D$, Plot of the threshold angle (mean $\pm \mathrm{SD}$ ) for target 3 versus target 1 in the same 10 preparations as in $B$. Same illustration conventions as in $C$. Threshold angles also cluster within $1 \mathrm{SD}$ of the diagonal, indicating no significant differences between the two conditions. lie within $1 \mathrm{SD}$ of the diagonal and therefore are not significantly different from one another. Figures $5 D$ and $6 D$ illustrate that the mean threshold angles were also not statistically different from those obtained in control experiments. Two mean threshold angles of 12 preparations lay $>1$ SD away from the diagonal, but $<2$ (Fig. 6D). Therefore the difference was not statistically significant at the $95.4 \%$ confidence level.

\section{Angular threshold computation and target approaching angle}

We investigated the effect of the angle of approach on LGMD/ DCMD firing by rotating the animal around a virtual vertical axis passing through the center of the stimulated eye. This allowed us to present looming targets from a wide range of approaching angles. Let $0^{\circ}$ be the position at which the animal's front faces the stimulation screen and positive angles denote counterclockwise rotation. If we consider the right eye, the angular range covered target approach angles from the back $\left(135^{\circ}\right)$ on the same side as the stimulated eye to $-45^{\circ}$ on the contralateral side (Fig. $7 A$, inset).

Figure $7 A$ illustrates an experiment in which the animal was stimulated at $0^{\circ}(\boldsymbol{O}$, facing the screen $)$ and at $90^{\circ}(\triangle$, perpendicular to the screen). No statistical differences were observed in the timing of the peak firing rate under these two conditions. The variability in the time of peak firing rate, however, usually increased from $90^{\circ}$ to $0^{\circ}$. In Figure $7 A$ for example, the largest SDs in the peak firing time at each value of $l /|v|$ were in all but one case $(l /|v|=20 \mathrm{msec})$ attributable to stimulation at $0^{\circ}$. Figure $7 B$ summarizes the results of 5 similar experiments of 13 that had the most reliable responses at $0^{\circ}$. The mean angular thresholds and delays under these two experimental conditions are plotted together with their SDs, and experiments performed on the same animal are connected by a dotted line. There were no statistical differences between the mean delay values in all cases. In one experiment (see $\triangle$, top left), the mean threshold angles were different at the $95.4 \%$ level, whereas the remaining threshold angles showed no statistically significant differences (one mean threshold angle, indicated by an arrow, lay within 2 SDs of its control experiment and was therefore not statistically different at the $95.4 \%$ confidence level). Here also, in all but one case, variability increased from $90^{\circ}$ to $0^{\circ}$. This increase in variability was correlated with the number of action potentials elicited per stimulus presentation at various approach angles. Figure $7 C$ plots for one experiment the mean number of action potentials $( \pm \mathrm{SD})$ elicited at three values of $l /|v|$ for looming black squares with approach angles $135^{\circ}, 90^{\circ}, 45^{\circ}$, and $0^{\circ}$. As in Figure $7, A$ and $B$, no statistically significant differences were observed in the timing of the peak firing rate in all cases analyzed $(n=5)$. Furthermore, the number of action potentials elicited per trial was remarkably constant over a large portion of the visual field ranging from $135^{\circ}$ to $45^{\circ}$. Between $45^{\circ}$ and $0^{\circ}$, however, the response declined abruptly, by $>50 \%$ in this experiment; similar results were obtained in the remaining four experiments. In the experiment illustrated in Figure $7 C$, there was a clear decrease in the number of action potentials elicited per trial as $l /|v|$ decreased from 50 to 
Figure 6. The linear relationship between time of peak firing rate and $l /|v|$ remains unchanged when several edges are added to the looming $\operatorname{target} A$, Plot of the peak firing rate as a function of $l /|v|$ for a square target (target 1: $\mathbf{\square}, \alpha=2.7 \pm$ $0.5, \delta=8.7 \pm 7.9 \mathrm{msec}$ ) and a textured target consisting of four concentric squares (target $4: \triangle$, $\alpha=3.5 \pm 0.8, \delta=18.5 \pm 10.2 \mathrm{msec}$ ) measured in the same preparation (L. migratoria). For clarity, only the largest SD is shown in one direction for each value of $l|| v \mid . B$, Plot of the mean delay for target $1(\square)$ and target $4(\triangle)$ versus mean angular threshold in 12 different preparations ( 9 $S$. americana and 3 L. migratoria). Experiments performed on the same preparation are connected by a dashed line. $C$, Plot of the delay (mean $\pm \mathrm{SD}$ ) for target 4 versus the delay for target 1 in the same 12 preparations as in $B$. For clarity, SDs are illustrated in one direction only. The dashed diagonal line represents identity. Delays cluster within $1 \mathrm{SD}$ of the diagonal, indicating no significant differences between the two conditions. $D$, Plot of the threshold angle (mean $\pm \mathrm{SD}$ ) for target 4 versus target 1 in the same 12 preparations as in $B$. Same illustration conventions as in $C$. Threshold angles cluster within $1 \mathrm{SD}$ of the diagonal, indicating no significant differences between the two conditions.
A

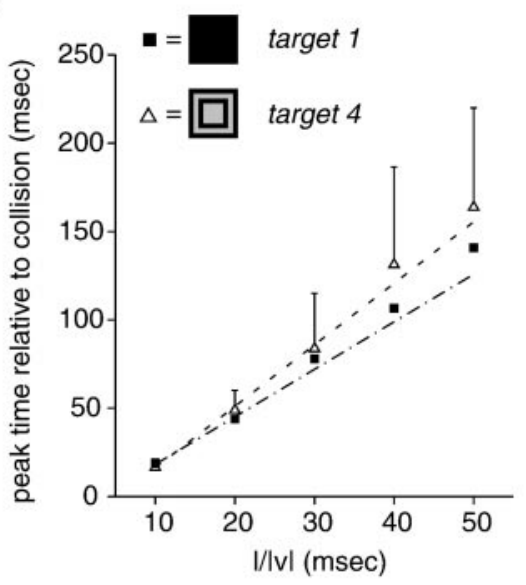

\section{C}

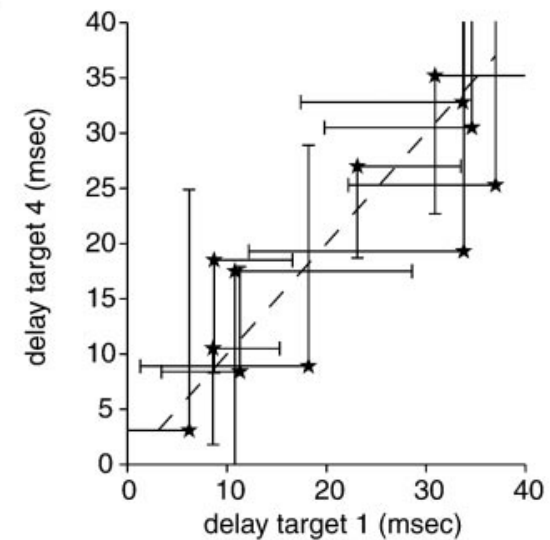

B
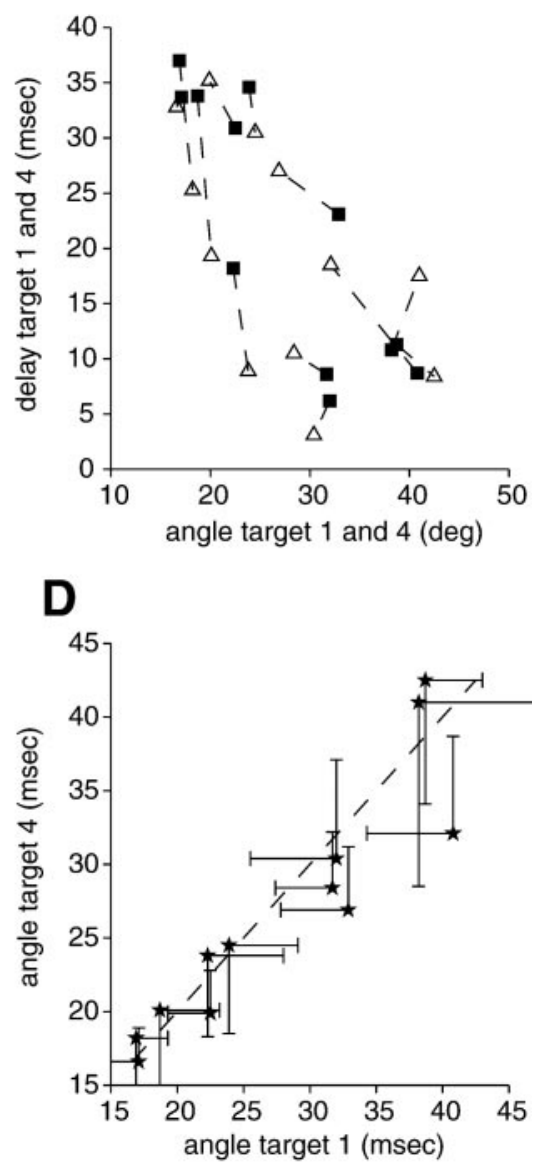

10 msec. However, this observation could not always be made so clearly on other preparations (Gabbiani et al., 1999a).

This decrease in the number of spikes per trial produced at $0^{\circ}$ was compensated for by the contralateral DCMD. Figure $7 D$ illustrates the number of action potentials $( \pm S D)$ recorded in each of the two DCMDs during an experiment in which the target approaching angle was varied between 5 values in the frontal visual field: $45^{\circ}, 22.5^{\circ}, 0^{\circ},-22.5^{\circ}$, and $-45^{\circ}$. The response recorded in the left connective $(\star)$ decreased from $45^{\circ}$ to $0^{\circ}$, as in Figure $7 C$, and essentially vanished at $-45^{\circ}$. Concomitantly, the number of action potentials recorded from the right connective (O) increased over the same range, resulting in the total number of action potentials elicited from both DCMDs per trial $(\triangle)$ being constant over the entire range $\left(-45^{\circ}-45^{\circ}\right)$. Similar results were obtained in $n=11$ additional animals.

\section{Two-dimensional linear summation model for motion- dependent excitation and size-dependent inhibition}

The experimental results described above were compared with those obtained in a two-dimensional model of the responses of the LGMD to looming stimuli. The motion-dependent excitatory input of the model was obtained by integrating the logarithm of the optic flow along the luminance edges of the expanding object weighted by a factor depending on the distance of the local motion stimulus to the central axis of the eye (Figs. $2 A-C$ and Eqs. 4 and 5). When the approaching object contained additional luminance edges, such as the CBP and the CSP described above, excitatory inputs were summed linearly for all luminance edges. The size-dependent inhibitory input was obtained at each time point by computing the surface covered by the object on the retina and transforming it by using the nonlinear function plotted in Figure $2 D$. Figure $8, A$ and $B$, illustrates the time course of excitation and inhibition for a disc approaching perpendicular to the body axis toward the center of the eye. The excitatory and inhibitory terms were subtracted from each other (Fig. 8C), and the times of peak firing rate were computed and plotted as a function $l /|v|$ (Fig. $8 D, 0$ ). As may be seen from this Figure, the prediction of the model is a linear function of $l /|v|$, although the slope of the linear relation is slightly smaller (4.69) than the prediction of the one-dimensional model [dashed line; slope identical to $\alpha=5$ ) of Gabbiani et al. (1999a)]. This, in turn, corresponds to a slightly larger threshold angle $\left(24^{\circ}\right.$ vs $\left.22^{\circ}\right)$. The difference, however, is well within the uncertainty of the experimental estimates for these parameters (Fig. $4 B, D$ ). As proven in Appendix 1, the two-dimensional model reduces exactly to the one-dimensional model for a looming disc if a less realistic choice of parameters is made, corresponding to circular iso-contour lines for the weight factor ( $a=1$ in Eq. 5) and no weight saturation beyond the optical resolution of the eye $\left(1 / 2 \theta_{\text {cutoff }}=0^{\circ}\right.$ in Eq. 5$)$.

Figure 9 summarizes the results for the remaining stimuli used in our experiments. As may be seen by comparing Figure $9 A$ with Figure $8 D$, the time of peak firing rate for a square is closer to the prediction of the one-dimensional model, and accordingly the slope is higher (4.86) corresponding to a smaller threshold angle $\left(23^{\circ}\right)$. These results are consistent with those observed experimentally, although the difference in threshold angles in the model $\left(\sim 1^{\circ}\right)$ is smaller than the mean 
A

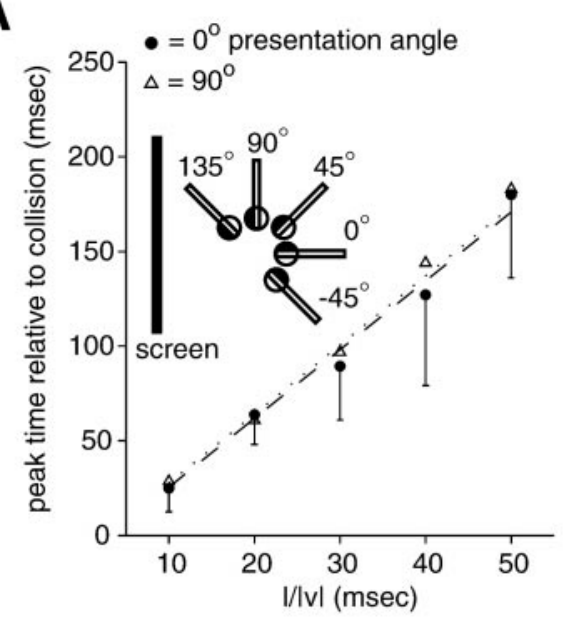

C

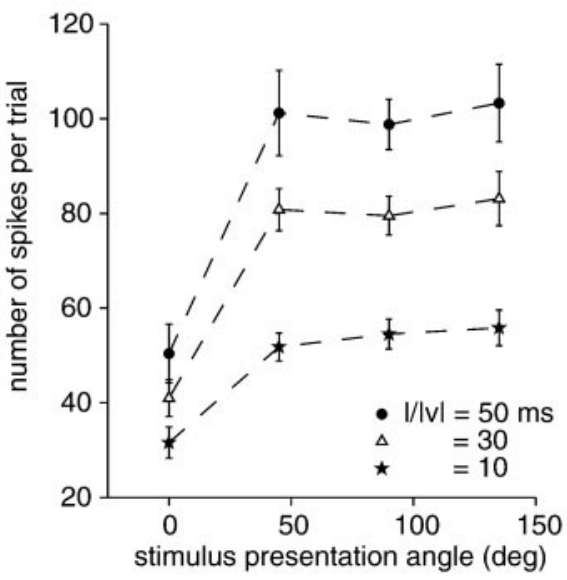

B

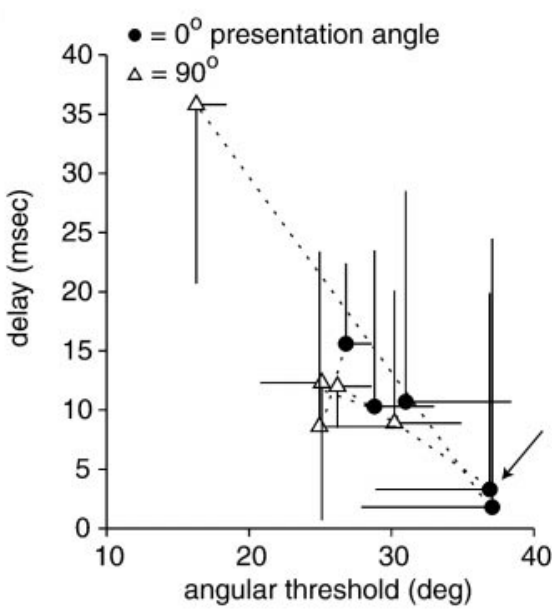

D

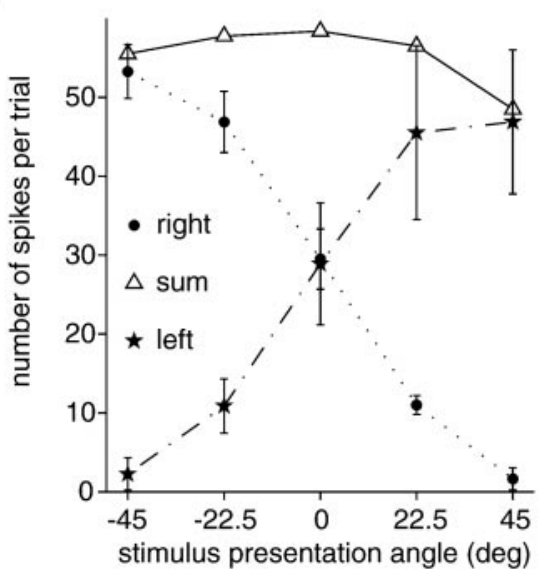

Figure 7. The linear relationship between time of peak firing rate and $l /|v|$ is invariant over a wide range of object approach angles, whereas the number of spikes decreases with presentation angle. $A$, Plot of the peak firing rate as a function of $l /|v|$ (mean $\pm \mathrm{SD}$ ) for a target approaching from the front $\left(0^{\circ}: \mathbf{0}, \alpha=\right.$ $3.6 \pm 0.9, \delta=10.7 \pm 17.8 \mathrm{msec})$ and for a target approaching from the side $\left(90^{\circ}: \triangle, \alpha=\right.$ $3.7 \pm 0.6, \delta=8.9 \pm 11.2 \mathrm{msec}$ ). For clarity, only the largest SD in one direction is shown at each value of $l /|v|$. Inset, Schematic diagram illustrating the definition of the approach angle with respect to the screen and the animal. Counterclockwise rotation from $0^{\circ}$ defines positive angles. $B$, Plot of the delay versus angular threshold (mean $\pm \mathrm{SD}$ ) for the same two approaching directions as in $A$, measured in five preparations (S. americana). Experiments performed on the same animal are connected by a dotted line. Solid lines illustrate $\mathrm{SD}$ in one direction only for clarity. The extensive overlap of SDs (with one exception) for experiments performed on the same animal indicate no significant differences between the two stimulation conditions. Arrow, Angle that lies at $>1$, but $<2$ SD of its control. $C$, Number of spikes (mean $\pm \mathrm{SD}$ ) elicited per trial as a function of presentation angle at three different values of $l /|v|(50 \mathrm{msec}$ : -; 30 msec: $\triangle$; 10 msec: $\star$ ) measured in the same preparation. $D$, Number of spikes (mean \pm SD) elicited as a function of presentation angle recorded simultaneously from both DCMDs, the axons of which run in the right $(\bullet)$ and the left $(\star)$ connectives $(l /|v|=$ $30 \mathrm{msec}$ ). The sum of both mean spike counts $(\triangle)$ is approximately independent of the presentation angle. value derived from Figure $4 D\left(\sim 3^{\circ}\right)$. In the model, the earlier peak firing time for a square stimulus versus a disc is not surprising: as explained in Results, the optic flow associated with a square is smaller than that of the corresponding disc and its surface is larger. Consequently, inhibition will start to dominate the excitatory term earlier, leading to earlier peak times.

Figure 9, $B$ and $C$, illustrates the results for the CBP and the CSP patterns: the time of peak firing still varies linearly with $l /|v|$, but as the strength of the excitatory term is increased by the additional moving edges in the stimulus, the timing of the peak moves closer to collision time. The values of the slopes in these two cases (1.56 and 0.92) correspond to predicted threshold angles of $65^{\circ}$ and $95^{\circ}$, respectively, that are incompatible with the experimental observations of Figures 5 and 6. Similarly, presentation of square targets approaching at different angles disrupted significantly the predictions of the model for deviations as small as $15^{\circ}$ with respect to the $90^{\circ}$ approach direction (Fig. 9D) (the slope equals 3.73 and the value of the threshold angle is $30^{\circ}$ ). Approach angles of $45^{\circ}$ and $0^{\circ}$, such as those used experimentally, could not be explained by the linear model (slopes of 1 and 0 corresponding to threshold angles of $90^{\circ}$ and $180^{\circ}$, respectively).

\section{DISCUSSION}

Earlier results have shown that the peak firing rate of LGMD/ DCMD always occurs at a fixed delay after a solid black square looming toward the animal (perpendicular to the body axis) has reached a threshold angle (or size) on the locust's retina, regard- less of the actual size of the square or speed of approach (Gabbiani et al., 1999a). Building on this work, we investigated in three locust species whether this angular threshold computation depends on stimulus changes expected to alter the spatial and temporal activation of inputs onto the dendritic tree of the LGMD. The results indicate that this angular threshold computation remains invariant under a wide range of experimental conditions. Furthermore, this invariance is not compatible with linear summation of excitatory and inhibitory inputs within the dendritic tree of LGMD.

\section{Identical computation implemented in three locust species}

Our initial observations were based on experiments with $S$. americana. Here, they were generalized to two African locust species, $S$. gregaria and L. migratoria. $S$. gregaria resembles very closely $S$. americana, but hybridization studies have shown it to be a distinct species (Harvey, 1981; Jago et al., 1979). It is the only Old World species of the Schistocerca genus that has richly radiated to comprise at least 42 species in the New World (Otte and Nasrecki, 1997). The 42 New World species are thought to have evolved from $S$. gregaria after one or more crossings of the Atlantic Ocean (Ritchie and Pedgley, 1989; Amedegnato, 1993). In contrast, L. migratoria belongs to a different subfamily of Acridids (Otte and Nasrecki, 1997) that evolved independently for $>100$ million years (Vickery, 1987; Whitington and Bacon, 1999). Therefore, angular threshold detection might be an escape mechanism common to many other orthopteran species (Rowell, 
A

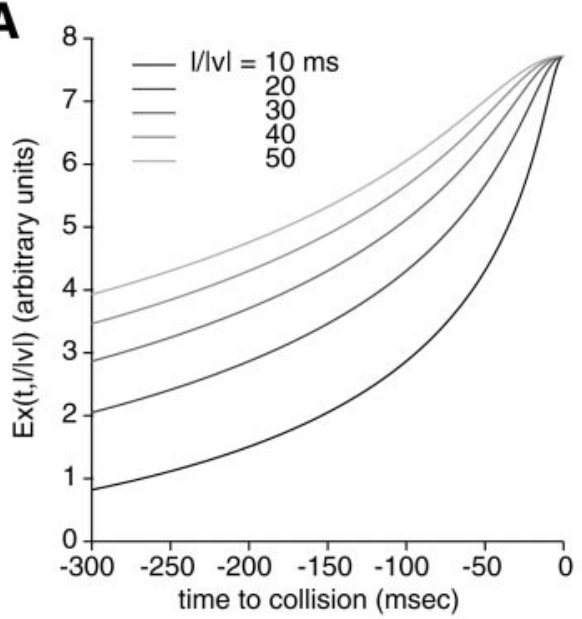

Figure 8. Model response to a disc looming toward the animal along a trajectory perpendicular to the body axis. $A$, Time course of excitation before collision for the five values of $l /|v|$ used experimentally. $B$, Time course of inhibition before collision. $C$, Model output obtained by linear combination of excitation and inhibition. $D$, Peak time (obtained from model, $C)$ as a function of $l /|v|$. The dashed line is the prediction of the one-dimensional model that matches experimental data.
B
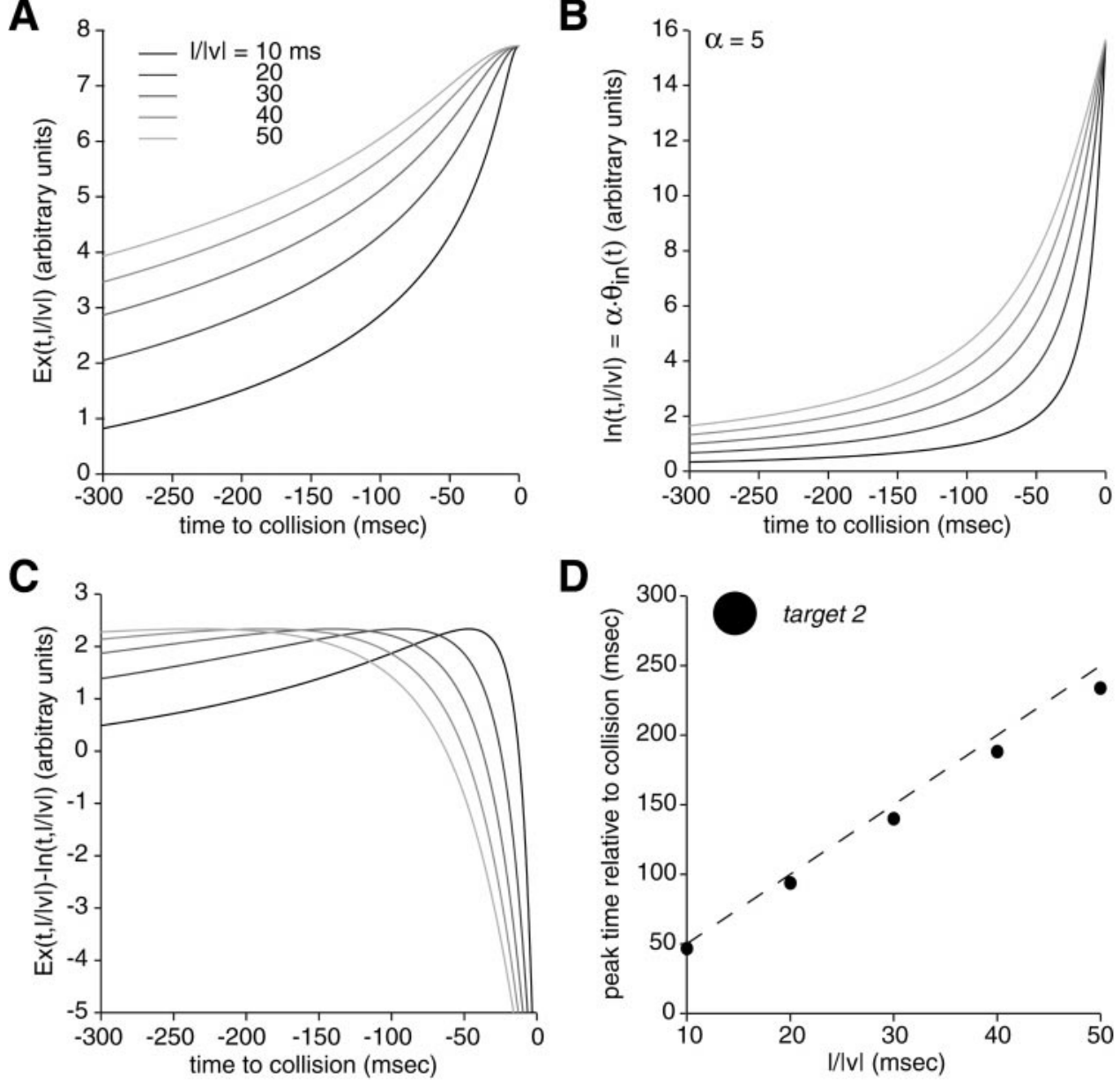

D

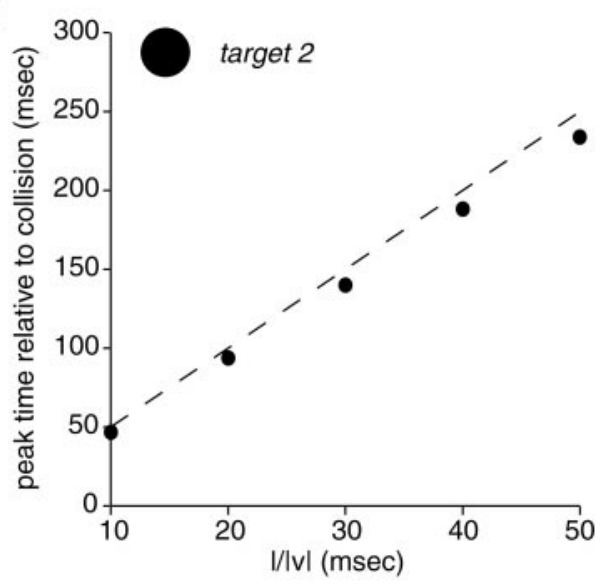

1971). Sun and Frost (1998) recast this hypothesis in a broader context by showing that a class of neurons in the nucleus rotundus of the pigeon have response properties identical to those of LGMD/DCMD neurons. Whether similar responses also arise in mammalian neurons remains to be investigated (Luksch et al., 1998). Finally, our results rule out interspecies differences as an explanation for the results reported by Rind and Simmons (1997) [see Gabbiani et al. (1999a,b) for a complete discussion].

\section{Invariance to target shape}

We investigated the effect of target shape on LGMD/DCMD responses by comparing the timing of the peak firing rate during the approach of circular and square targets. The difference between these two stimuli is detectable in principle when the objects cover nine ommatidia or $4^{\circ}$ in visual angle on the retina, that is, before LGMD and DCMD begin to respond during approach. However, simulation results using the two-dimensional model of the responses of the LGMD suggested that only a small decrease $\left(\sim 1^{\circ}\right)$ in the angular threshold size should be observed for a square target with respect to a circular target, because of a decrease of optic flow stimulation and an increase of sizedependent inhibition. This is in agreement with experimental observations that point to a $\sim 3^{\circ}$ decrease in average angular threshold size for square targets. This change lies within the margin of accuracy of our measurements.

\section{Invariance to target texture}

In contrast, the two textured targets used in the present experiments contain inner edges that may be expected to result in a substantial additional spread of excitation over the time course of approach. The two-dimensional model of the responses of the LGMD demonstrates that linear summation of the excitation attributable to inner luminance edges in the CBP and CSP significantly alters the peak firing rate time for both targets. Such changes, however, were not observed experimentally. A possibility is that these additional excitatory inputs are filtered out presynaptically to LGMD. This appears unlikely: the contrast of the CBP and CSP were in the range for which LGMD/DCMDs are known to respond vigorously to expanding objects, thus ruling out early gain control mechanisms. In the case of the CSP (and to a lesser extent of the CBP), one might argue that excitatory inputs triggered by the outer object contour/edge might have caused a strong decrement in the response to inner edges, because the same ommatidia were stimulated successively within a brief interval. However, repeated local stimulation with moving targets showed that habituation to moving stimuli occurs much more slowly than could occur with the present stimuli (Krapp and Gabbiani, 2000). Finally, lateral inhibition is unlikely to be effective in the case of the CSP (and presumably played no role for the CBP) because successive edges were separated by more than one to two interommatidial angles over most of the approach trajectory (as soon as the object subtended $16^{\circ}$ in visual angle, assuming an interommatidial angle of $2^{\circ}$ ) (Horridge, 1978).

\section{Invariance to target approach angle}

When the angle of target approach was varied, the timing of the peak LGMD/DCMD firing rate remained invariant over $135^{\circ}$ on 

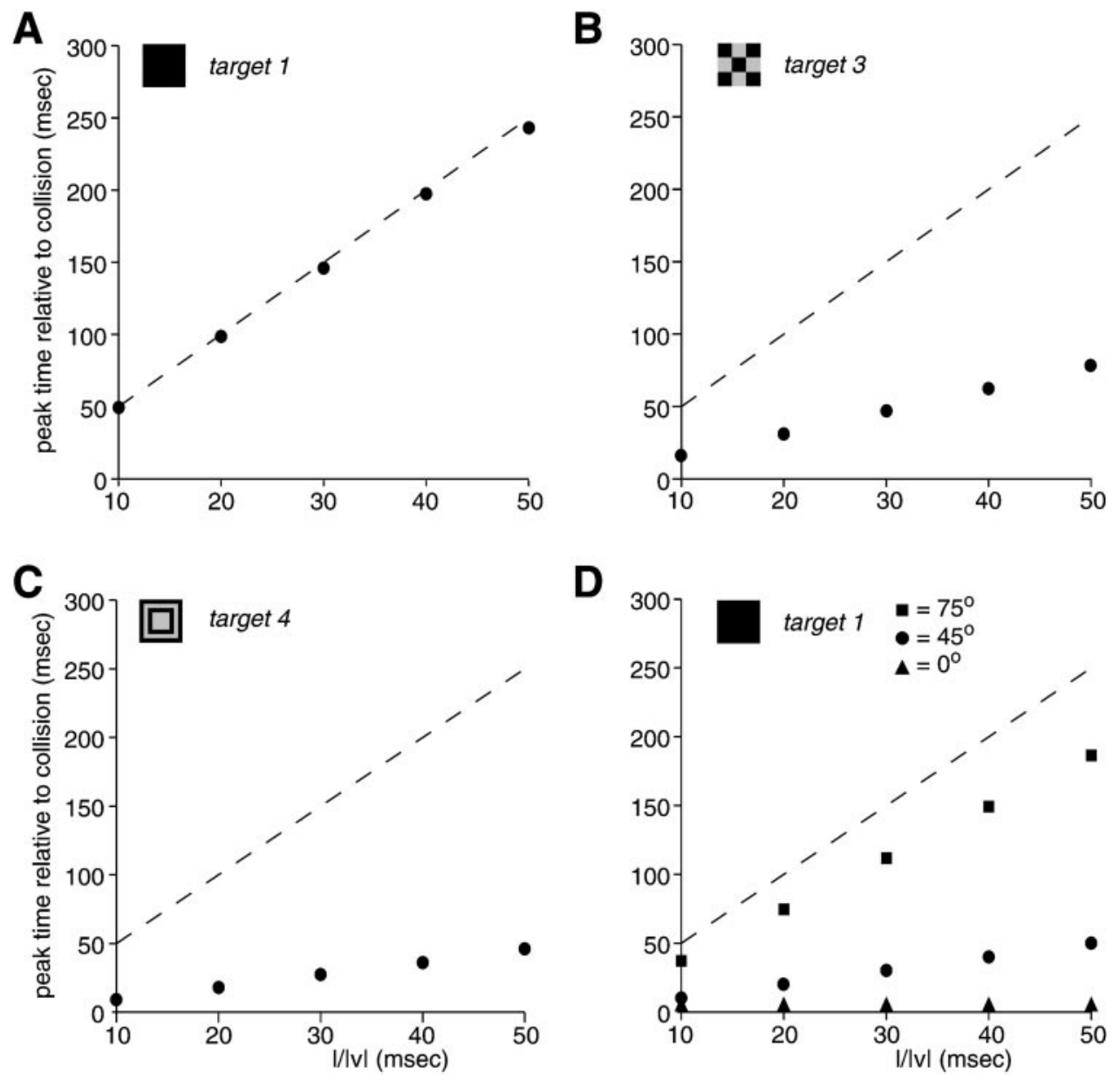

Figure 9. Peak time (model) as a function of $l /|v|$ for the various experimental targets. $A$, Square target. $B$, CBP. $C$, CSP. $D$, Solid square approaching at three different angles $\left(75^{\circ}, 45^{\circ}\right.$, and $\left.0^{\circ}\right)$ measured from the eye center in the horizontal plane. In $A-D$, the dashed line is the prediction of the onedimensional model that matches experimen-

one side of the animal's longitudinal body axis. Both pairs of LGMD/DCMDs thus provide an invariant warning signal to potentially threatening objects looming toward the animal over at least $270^{\circ}$ in the horizontal plane. This is remarkable because the temporal and spatial activation sequence of inputs onto the dendritic tree of the LGMD may be expected to differ completely across these conditions. For an object approaching at $90^{\circ}$, the expansion will be symmetric around the center of the eye, whereas at $135^{\circ}$ the expansion is maximally asymmetric, with one vertical edge sweeping over most of the receptor array. In accordance with this, the two-dimensional model of LGMD predicted non-invariant responses over the range of approach angles tested experimentally.

In the frontal visual field, responses decreased rapidly as the approach angle shifted toward the contralateral side. This was presumably caused by two factors: (1) the ommatidia sample only a limited portion of the contralateral visual field $\left(\sim 10^{\circ}\right)$, implying that the vertical edge of a looming square opposite to the eye is positioned outside of the receptive field of the LGMD over most of a frontal approach and cannot excite the neuron; (2) the sensitivity to moving targets is weaker in the frontal visual field than at the center of the eye (Rowell, 1971; Krapp and Gabbiani, 2000). This decrease in DCMD response leads in turn to an increase in peak firing time variability that could conceivably be reduced by averaging over both responses of the DCMDs because they are independent. Such a procedure is plausible, given the relatively constant number of action potentials produced by both DCMDs over the frontal field of view. Alternatively, preparation for escape in natural conditions might involve repositioning of the animal with respect to the target (Hassenstein and Hustert, 1999).

Robertson and Gray (1997) first recorded the responses of both DCMDs to frontal targets in tethered flying locusts. On the basis of behavioral studies (Robertson and Johnson, 1993), they suggested that the relative number of action potentials in both DCMDs might trigger directional steering maneuvers during flight to avoid frontal obstacles. Our results confirm that both DCMDs can encode target approach direction (left vs right; see the SD in Fig. $7 D$ at $\pm 22.5^{\circ}$ ), although the increased variability of responses to frontal targets suggests that directional steering behaviors would be triggered outside of their optimal operating range. Accurate directional estimates may require additional signals, probably available from other lobula movement-sensitive neurons (Gewecke and Hou, 1993).

\section{Biophysical mechanisms responsible for invariance to target texture and approach angle}

One explanation for the observed texture response invariance could be nonlinear saturation of postsynaptic excitatory inputs. In addition, active membrane conductances might contribute to suppress the excitation expected from inner luminance edges. Invariance to approach angles could result from the geometric arrangement of excitatory inputs onto the dendrites of LGMD as well as passive attenuation and active amplification of contributions associated with specific positions in the visual field. To be tested rigorously, these hypotheses will require a precise mapping of the excitatory retinotopic inputs onto the dendritic tree of the 
LGMD, as done for wide-field tangential neurons in flies (Egelhaaf and Borst, 1995) and an assessment of the passive and active properties of the neuron.

\section{LGMD as a model for invariant feature extraction}

A major threat to survival for locusts and other insects in the wild is embodied by predatory birds. Species foraging on insects vary in shape, body texture, and predatory tactics. They will typically engage in chases from various positions with respect to their prey (Jablonski, 1999). It is therefore not surprising that locusts and other insects have evolved escape behaviors to confront these dangers. In the locust species investigated here, one can extract from the LGMD/DCMD peak firing time the angular threshold of an approaching object regardless of its shape, texture, or direction of approach. Because visual stimuli reaching each ommatidia on the locust's retina are thought to be first integrated temporally and spatially in the dendritic tree of the LGMD, our results suggest that this invariance is implemented within the LGMD dendrites. LGMD is uniquely identifiable from animal to animal and accessible to intracellular recordings; it therefore offers a model for the study of angular threshold computation and its biophysical implementation (Gabbiani et al., 1999a). The present study suggests that LGMD is also an ideal model for studying the emergence of invariant receptive field properties at the dendritic and biophysical levels.

\section{APPENDIX 1}

In this appendix we show that if $a=1$ and $1 / 2 \theta_{\text {cutoff }}=0^{\circ}$ in Equation 5, the two-dimensional model of Equations 3, 5, and 6 reduces to the one-dimensional model of Equation 2 for a circle approaching at an angle of $90^{\circ}$.

The spherical coordinate system of Figure $2 B$ corresponds to

$$
x=R \cos \vartheta, \quad y=R \sin \vartheta \cos \varphi, \quad z=R \sin \vartheta \sin \varphi,
$$

where $R$ is the radius of the eye. Let us denote by $S_{\mathrm{x}>0}^{2}$ the hemisphere used to model the eye. The scalar product induced on $S_{\mathrm{x}>0}^{2}$ by the three-dimensional Euclidean scalar product is described in spherical coordinates by the matrix:

$$
G=R^{2} \cdot\left(\begin{array}{cc}
1 & 0 \\
0 & \sin ^{2} \vartheta
\end{array}\right)
$$

(Dubrovin et al., 1991). The boundary of the circle on the retina is described by the equation:

$$
\begin{gathered}
\gamma:(-\infty ; 0) \times[0 ; 2 \pi) \rightarrow S_{\mathrm{x}>0}^{2} \\
(t, \varphi) \mapsto(\vartheta(t) \varphi)^{\mathrm{T}},
\end{gathered}
$$

with $\vartheta(t)=\tan ^{-1} l / v t$. The subscript $(\cdot)^{\mathrm{T}}$ denotes matrix transposition. We compute first the excitatory term:

$$
\operatorname{Ex}\left(t_{0}, \frac{l}{|v|}\right)=\frac{1}{2 \pi} \int_{[0 ; 2 \pi]} d l_{\gamma} \cdot w(\vartheta, \varphi)^{-1} \cdot \log _{+} \frac{1}{R}\left\langle\dot{\gamma}_{\mathrm{t}_{0}}(\varphi) ; \mathbf{n}_{\mathrm{t}_{0}}(\varphi)\right\rangle .
$$

Because $1 / 2 \theta_{\text {cutoff }}=0^{\circ}$, the second term in Equation 5 vanishes and the first term reduces to

$$
w(\vartheta, \varphi)=R \cdot|\sin \vartheta|
$$

where we have used $a=1$ and the trigonometric identity $\sin ^{2} \varphi+$ $\cos ^{2} \varphi=1$. The tangent vector to the curve $\gamma\left(t_{0}, \cdot\right)$ is given by:

$$
\gamma_{\mathrm{t}_{0}}^{\prime}(\varphi)=\frac{\partial}{\partial \varphi} \gamma\left(t_{0}, \varphi\right)=\left(\begin{array}{ll}
0 & 1
\end{array}\right)^{\mathrm{T}}
$$

and its norm by:

$$
\left\|\gamma_{\mathrm{t}_{0}}^{\prime}(\varphi)\right\|=\left\langle\gamma_{\mathrm{t}_{0}}^{\prime}(\varphi) ; \gamma_{\mathrm{t}_{0}}^{\prime}(\varphi)\right\rangle^{1 / 2}=\left(\gamma_{\mathrm{t}_{0}}^{\prime} \mathrm{T}(\varphi) \cdot G \cdot \gamma_{\mathrm{t}_{0}}^{\prime}(\varphi)\right)^{1 / 2}=R \cdot\left|\sin \vartheta\left(t_{0}\right)\right| .
$$

The line element in Equation A2 is given by:

$$
d l_{\gamma}(\varphi)=\left\|\gamma_{\mathrm{t}_{0}}^{\prime}(\varphi)\right\| \cdot d \varphi=R \cdot\left|\sin \vartheta\left(t_{0}\right)\right| \cdot d \varphi
$$

The expansion vector is given by:

$$
\dot{\gamma}_{\mathrm{t}_{0}}(\varphi)=\left(\begin{array}{ll}
\dot{\vartheta}\left(t_{0}\right) & 0
\end{array}\right)^{\mathrm{T}},
$$

and the outward pointing unit normal vector is easily seen to be equal to:

$$
\mathbf{n}_{\mathrm{t}_{0}}(\varphi)=\left(\begin{array}{ll}
\frac{1}{R} & 0
\end{array}\right)^{\mathrm{T}}
$$

because:

$$
\left\langle\mathbf{n}_{\mathrm{t}_{0}}(\varphi) ; \mathbf{n}_{\mathrm{t}_{0}}(\varphi)\right\rangle=\mathbf{n}_{\mathrm{t}_{0}}(\varphi)^{\mathrm{T}} \cdot G \cdot \mathbf{n}_{\mathrm{t}_{0}}(\varphi)=1,
$$

and

$$
\left\langle\mathbf{n}_{\mathrm{t}_{0}}(\varphi) ; \gamma_{\mathrm{t}_{0}}^{\prime}(\varphi)\right\rangle=\mathbf{n}_{\mathrm{t}_{0}}(\varphi)^{\mathrm{T}} \cdot G \cdot \gamma_{\mathrm{t}_{0}}^{\prime}(\varphi)=0 .
$$

We compute only the first term of Equation 4 (the second term is an easily calculated constant). Using Equations A5 and A6:

$$
\begin{array}{r}
\log \frac{1}{R}\left\langle\mathbf{n}_{\mathrm{t}_{0}}(\varphi) ; \dot{\gamma}_{\mathrm{t}_{0}}(\varphi)\right\rangle=\log \frac{1}{R}\left(\frac{1}{R} 0\right) \cdot R^{2}\left(\begin{array}{cc}
1 & 0 \\
0 & \sin ^{2} \vartheta\left(t_{0}\right)
\end{array}\right) \cdot\left(\begin{array}{c}
\dot{\vartheta}\left(t_{0}\right) \\
0
\end{array}\right) \\
=\log \dot{\vartheta}\left(t_{0}\right) . \quad(\mathrm{A} 7)
\end{array}
$$

Plugging Equations A3, A4, and A7 in A2 we deduce that:

$$
\begin{aligned}
\operatorname{Ex}\left(t_{0}, \frac{l}{|v|}\right)= & \frac{1}{2 \pi} \int_{0}^{2 \pi} d \varphi \cdot R\left|\sin \vartheta\left(t_{0}\right)\right| \cdot\left(R\left|\sin \vartheta\left(t_{0}\right)\right|\right)^{-1} \cdot \log \dot{\vartheta}\left(t_{0}\right) \\
& =\log \dot{\vartheta}\left(t_{0}\right)
\end{aligned}
$$

which matches the excitatory term of Equation 2, because $1 / 2 \dot{\theta}(t)=\dot{\vartheta}(t)$ for a circle approaching at $90^{\circ}$.

The surface covered by the disc on the eye at time $t_{0}$ is given by:

$$
\begin{aligned}
S\left(t_{0}\right) & =\int_{0}^{2 \pi} d \varphi \int_{0}^{\vartheta(\mathrm{t})} d \vartheta \cdot \sqrt{|\operatorname{det} G|} \\
& =2 \pi R^{2} \int_{0}^{\vartheta(\mathrm{t})} d \vartheta \cdot\left|\sin \vartheta\left(t_{0}\right)\right| \\
& =2 \pi R^{2}\left(1-\cos \vartheta\left(t_{0}\right)\right)
\end{aligned}
$$

(Dubrovin et al., 1991). Inverting this latter result yields:

$$
\vartheta(t)=\cos ^{-1}\left(1-\frac{S(t)}{2 \pi R^{2}}\right),
$$

and therefore $2 \vartheta(t)=\theta_{\text {in }}(t)$ for a sphere of radius 1 .

\section{APPENDIX 2}

In this appendix we give the formulas used to compute $\operatorname{Ex}(t, l /|v|)$ and $\operatorname{In}(t, l /|v|)$ for squares approaching from various angles with 
respect to the eye's central axis. These formulas were used to write the Maple scripts available at http://glab.bcm.tmc.edu (under the title of this article after following the Publication link).

\section{Top and bottom boundaries}

The three-dimensional approach trajectory of the top and bottom boundaries (Fig. $2 B$ ) of a square is described by:

$$
(t b) \mapsto(x(t) y(b) z)^{\mathrm{T}}=(v t b l \pm l)^{\mathrm{T}},
$$

where each value of $b \in[-1 ; 1]$ corresponds to a point on the square edge. The projection of the edge on the eye is given by:

$$
\begin{gathered}
\gamma:(-\infty ; 0) \times[-1 ; 1] \rightarrow S_{\mathrm{x}>0}^{2} \\
(t b) \mapsto(\vartheta(t, b) \varphi(b))^{\mathrm{T}},
\end{gathered}
$$

with

$$
\begin{gathered}
\varphi(b)= \pm \cot ^{-1} b, \\
\vartheta(t, b)=\tan ^{-1} \frac{l \sqrt{1+b^{2}}}{v t}=\tan ^{-1} \frac{\beta}{t} \sqrt{1+b^{2}},
\end{gathered}
$$

and where we have set $\beta=l / v$.

\section{Left and right boundaries}

With a notation similar to the one used above, the right and left square edges are described by

$$
(t b) \mapsto(x(t) \text { y } z(b))^{\mathrm{T}}=(v t \pm l b l)^{\mathrm{T}}
$$

(Fig. $2 B$ ). Their projection onto the eye surface is given by:

$$
\begin{gathered}
\gamma:(-\infty ; 0) \times[-1 ; 1] \rightarrow S_{\mathrm{x}>0}^{2} \\
(t b) \mapsto(\vartheta(t, b) \varphi(b))^{\mathrm{T}} .
\end{gathered}
$$

For the right boundary,

$$
\varphi(b)= \pm \cot ^{-1} \frac{1}{b} \quad \text { for } b \gtrless 0 \text {, respectively, }
$$

and

$$
\vartheta(t, b)=\tan ^{-1} \frac{\beta}{t} \sqrt{1+b^{2}}
$$

For the left boundary, $\varphi(b)$ is given by

$$
\varphi(b)= \pm \pi \mp \cot ^{-1} \frac{1}{b}, \quad \text { for } b \gtrless 0 \text {, respectively, }
$$

with $\vartheta(t, b)$ unchanged.

\section{Approach angles different from $90^{\circ}$}

For approach angles different from $90^{\circ}$ (not perpendicular to the body axis), the optic flow was computed in a coordinate system adapted to the approach direction (i.e., with its $x$-axis passing through the center of the square). The parametrization of the square edges given above therefore remained unchanged.

\section{Weight factor for arbitrary approach angles}

To compute the weight factor $w(\vartheta, \varphi)$, the coordinates of a point on the square edge with respect to the coordinate system whose $x$-axis passes through the eye center are needed (Fig. 2). Let $(x y z)^{\mathrm{T}}$ be the coordinates of the point in the coordinate system adapted to the approach direction (used to compute the optic flow component of excitation; see last paragraph) and let $\psi$ denote the angle of the approach direction with respect to the eye center (i.e., $\psi=0$ for an approach angle of $\left.90^{\circ}\right)$. Let $\left(x_{\psi} y_{\psi} z_{\psi}\right)^{\mathrm{T}}$ be the corresponding coordinates in the coordinate system whose $x$-axis passes through the eye center. The values of $\left(x_{\psi} y_{\psi} z_{\psi}\right)^{\mathrm{T}}$ are obtained by rotating $(x y z)^{\mathrm{T}}$ by an angle $\psi$ about the $z$-axis,

$$
\left(\begin{array}{l}
x_{\psi} \\
y_{\psi} \\
z_{\psi}
\end{array}\right)=\left(\begin{array}{ccc}
\cos \psi & -\sin \psi & 0 \\
\sin \psi & \cos \psi & 0 \\
0 & 0 & 1
\end{array}\right)\left(\begin{array}{l}
x \\
y \\
z
\end{array}\right) .
$$

We next project the point onto the surface of the eye:

$$
\left(\begin{array}{c}
x_{\psi} \\
y_{\psi} \\
z_{\psi}
\end{array}\right) \rightarrow \frac{R}{D}\left(\begin{array}{c}
x_{\psi} \\
y_{\psi} \\
z_{\psi}
\end{array}\right)
$$

with $D=\sqrt{x_{\psi}^{2}+y_{\psi}^{2}+z_{\psi}^{2}}=\sqrt{x^{2}+y^{2}+z^{2}}$ and compute the weighted distance to the eye center,

$$
w(\vartheta, \varphi)=\frac{R}{D}\left(\frac{y_{\psi}^{2}}{a^{2}}+a^{2} z_{\psi}^{2}\right)^{1 / 2} .
$$

For the bottom and top boundaries, we have at time $t$ for the point on the edge corresponding to the parameter $b$,

$$
D=l \sqrt{\frac{t^{2}}{\beta^{2}}+b^{2}+1},
$$

and

$$
\sqrt{\frac{y_{\psi}^{2}}{a^{2}}+a^{2} z_{\psi}^{2}}=l \sqrt{\frac{1}{a^{2}}\left(\frac{t}{\beta} \sin \psi+b \cos \psi\right)^{2}+a^{2}},
$$

so that

$w(\vartheta(t, b), \varphi(b))=\frac{R}{\sqrt{\frac{t^{2}}{\beta^{2}}+b^{2}+1}} \sqrt{\frac{1}{a^{2}}\left(\frac{t}{\beta} \sin \psi+b \cos \psi\right)^{2}+a^{2}}$.

A similar calculation for the right and left boundaries yields, respectively,

$w(\vartheta(t, b), \varphi(b))$

$$
=\frac{R}{\sqrt{\frac{t^{2}}{\beta^{2}}+b^{2}+1}} \sqrt{\frac{1}{a^{2}}\left(\frac{t}{\beta} \sin \psi \pm \cos \psi\right)^{2}+b^{2} a^{2}} .
$$

For approach trajectories with angles $0^{\circ}<\psi<90^{\circ}$ the time $t_{\text {thres }}$ at which the left edge of the object reaches the eye boundary is given by $\tan \psi=v t_{\text {thres }} / l$, or, equivalently, $t_{\text {thres }}=\beta \tan \psi$. The portion of the top and bottom boundaries remaining on the eye after $t_{\text {thres }}$ is given by $b<b_{\text {thres }}(t), b_{\text {thres }}(t)=t / \beta \tan \psi>0$. For the approach trajectory $\psi=90^{\circ}, t_{\text {thres }}=-\infty$ and $b_{\text {thres }}=0$. Finally, for $\psi>\pi / 2 \operatorname{rad}\left(90^{\circ}\right), t_{\text {thres }}=\beta / \tan (\psi-(\pi / 2))$, and

$$
b_{\text {thres }}(t)=\frac{-t}{\beta \tan \left(\psi-\frac{\pi}{2}\right)}<0 .
$$

\section{Surface for arbitrary approach angles}

To compute the surface of the square projecting on the retina for arbitrary approach angles, we use the following spherical coordinate system:

$$
x=R \cos \vartheta \cos \varphi, \quad y=R \cos \vartheta \sin \varphi, \quad z=R \sin \vartheta,
$$


with the $x$-axis passing through the center of the approaching square. The surface element is given by $\sqrt{|\operatorname{det} G|}=R^{2}|\cos \vartheta|$ (Dubrovin et al., 1991), and the surface itself is given by:

$$
S(t)=\int_{\varphi_{\min }}^{\varphi_{\max }} d \varphi \int_{-\vartheta_{\mathrm{bound}}(\mathrm{t}, \varphi)}^{\vartheta_{\mathrm{bound}}(\mathrm{t}, \varphi)} d \vartheta \cdot R^{2} \cdot|\cos \vartheta|
$$

We distinguish the following cases: (1) for $\psi \leq 90^{\circ}$ and $t \leq t_{\text {thres }}$ : $\varphi_{\max / \min }= \pm \tan ^{-1} \beta / t$ and $\vartheta_{\text {bound }}(t, \varphi)=\tan ^{-1}(\beta / t \cos \varphi) ;(2)$ for $\psi \leq 90^{\circ}$ and $t \geq t_{\text {thres }}: \varphi_{\max }=\pi / 2-\psi$ and $\varphi_{\min }, \vartheta_{\text {bound }}$ are given by the above formulas; (3) for $\psi \geq 90^{\circ}$ and $t \leq t_{\text {thres }}: S(t)=$ 0 ; (4) for $\psi \geq 90^{\circ}$ and $t \geq t_{\text {thres }}: \varphi_{\max }=-(\psi-\pi / 2)$, $\varphi_{\min }=$ $-\tan ^{-1} \beta / t$ and $\vartheta_{\text {bound }}$ is as above.

\section{Checkerboard pattern}

It is easy to see that the projection of the horizontal and vertical inner edges of the checkerboard pattern on the eye may be parametrized with similar formulas as the outer edges. The top and bottom edges are given by:

$$
\begin{gathered}
\gamma:(-\infty ; 0) \times[-1 ; 1] \rightarrow S_{\mathrm{x}>0}^{2} \\
(t b) \mapsto(\vartheta(t, b) \varphi(b))^{\mathrm{T}}
\end{gathered}
$$

with

$$
\begin{gathered}
\varphi(b)= \pm \cot ^{-1} b / 3 \\
\vartheta(t, b)=\tan ^{-1} \frac{l \sqrt{(1 / 3)^{2}+b^{2}}}{v t}=\tan ^{-1} \frac{\beta}{3 t} \sqrt{1+(3 b)^{2}} .
\end{gathered}
$$

A similar formula holds for the left and right vertical edges. The corresponding excitatory term $\operatorname{Ex}_{\text {inner }}(t, l / 3 \cdot|v|)$ is obtained as usual by computing symbolically the integrand of Equation 3 and subsequent numerical integration.

\section{REFERENCES}

Amedegnato C (1993) African-American relationships in the Acridians (Insecta, Orthoptera). In: The Africa-South America connexion (George W, Lavocat R, eds), pp 59-75. Oxford: Oxford UP.

Bevington PR, Robinson DK (1992) Data reduction and error analysis for the physical sciences, Ed 2. Boston: McGraw-Hill.

Cavanagh P (1978) Size and position invariance in the visual system. Perception 7:167-177.

Dubrovin BA, Fomenko AT, Novikov SP (1991) Modern geometrymethods and applications, Part I: The geometry of surfaces, transformation groups and fields, Ed 2. Berlin: Springer.

Egelhaaf M, Borst A (1995) Calcium accumulation in visual interneurons of the fly: stimulus dependence and relationship to membrane potential. J Neurophysiol 73:2540-2552.

Forsythe GE, Malcom MA, Moler CB (1977) Computer methods for mathematical computations. Englewood Cliffs, NJ: Prentice Hall.

Gabbiani F, Krapp HG, Laurent G (1999a) Computation of object approach by a wide-field, motion-sensitive neuron. J Neurosci 19:1122-1141.

Gabbiani F, Hatsopoulos N, Krapp HG, Laurent G (1999b) The many ways of building collision-sensitive neurons. Trends Neusci 22:437-438.

Galarreta M, Hestrin S (1998) Frequency-dependent synaptic depression and the balance of excitation and inhibition in the neocortex. Nat Neurosci 1:587-594.

Gewecke M, Hou T (1993) Visual brain neurons in Locusta migratoria. In: Sensory systems of arthropods (Wiese K, Gribakin FG, Popov AV, Renninger G, eds), pp 119-144. Basel: Birkhäuser.

Harvey AW (1981) A reclassification of the Schistocerca americana complex (Orthoptera: Acrididae). Acridida 10:61-77.

Hassenstein B, Hustert R (1999) Hiding responses of locusts to approaching objects. J Exp Biol 202:1701-1710.

Hatsopoulos N, Gabbiani F, Laurent G (1995) Elementary computation of object approach by a wide-field visual neuron. Science 270:10001003.

Hildreth EC, Koch C (1987) The analysis of visual motion: from computational theory to neuronal mechanisms. Annu Rev Neurosci 10:477-533.

Horridge GA (1978) The separation of visual axes in apposition compound eyes. Philos Trans R Soc Lond B Biol Sci 285:1-59.

Jablonski PG (1999) A rare predator exploits prey escape behavior: the role of tail-fanning and plumage contrast in foraging of the painted redstart (Myioborus pictus). Behav Ecol 10:7-14.

Jago ND, Antoniou A, Scott P (1979) Laboratory evidence showing the separate species status of Schistocerca gregaria, americana and cancellata (Acrididae, Crytacanthacridinae). Syst Entomol 4:133-142.

Killmann F, Gras H, Schürmann F-W (1999) Types, numbers and distribution of synapses on the dendritic tree of an identified visual interneuron in the brain of the locust. Cell Tissue Res 296:645-665.

Krapp HG, Gabbiani F (2000) Spatial integration of local motion stimuli by a wide-field looming-sensitive neuron. Soc Neurosci Abstr 26:173.

Laurent G, Davidowitz H (1994) Encoding of olfactory information with oscillating neural assemblies. Science 265:1872-1875.

Luksch H, Cox K, Karten HJ (1998) Bottlebrush dendritic endings and large dendritic fields: motion-detecting neurons in the tectof ugal pathway. J Comp Neurol 396:399-414.

Olshausen B, Anderson CH, Van Essen DC (1993) A neurobiological model of visual attention and invariant pattern recognition based on dynamic routing of information. J Neurosci 13:4700-4719.

O'Shea M, Williams JLD (1974) The anatomy and output connection of a locust visual interneurone; the lobula giant movement detector (LGMD) neurone. J Comp Physiol 91:257-266.

Otte D, Naskrecki P (1997) Orthoptera species online. http://viceroy.eeb. uconn.edu/Orthoptera (03/03/00).

Palka J (1967) An inhibitory process influencing visual responses in a fibre of the ventral nerve cord of locusts. J Insect Physiol 13:235-248.

Pearson KG, Heitler WJ, Steeves JD (1980) Triggering of locust jump by multimodal inhibitory interneurons. J Neurophysiol 43:257-278.

Press WH, Teukolsky SA, Vetterling WT, Flannery BP (1992) Numerical recipies in C, Ed 2. Cambridge: Cambridge UP.

Reichardt W, Egelhaaf M, Schlögl RW (1988) Movement detectors provide sufficient information for local computation of 2-D velocity field. Naturwissenschaften 75:313-315.

Riesenhuber M, Poggio T (1999) Hierarchical models of object recognition in cortex. Nat Neurosci 2:1019-1025.

Rind FC (1984) A chemical synapse between two motion detecting neurons in the locust brain. J Exp Biol 110:143-167.

Rind FC, Simmons PJ (1992) Orthopteran DCMD neuron: a reevaluation of responses to moving objects. I. Selective responses to approaching objects. J Neurophysiol 68:1654-1682.

Rind FC, Simmons PJ (1997) Signaling of object approach by the DCMD neuron of the locust. J Neurophysiol 77:1029-1033.

Ritchie M, Pedgley D (1989) Desert locusts cross the Atlantic. Antenna 13:10-12.

Roberston RM, Gray JR (1997) Looming detector activity associated with flight steering in the locust. Soc Neurosci Abstr 23:767.

Roberston RM, Johnson AG (1993) Retinal image size triggers obstacle avoidance in flying locusts. Naturwissenschaften 80:176-178.

Roberston RM, Pearson KG (1983) Interneurons in the flight system of the locust: distribution, connections, and resetting properties. J Comp Neurol 215:33-50.

Rolls ET, Baylis GC (1986) Size and contrast have only small effects on the responses to faces of neurons in the cortex of the superior temporal sulcus of the monkey. Exp Brain Res 65:38-48.

Rowell CHF (1971) The orthopteran descending movement detector (DMD) neurons: a characterization and review. Z Vgl Physiol 73:167-194.

Rowell CHF, O'Shea M, Williams JLD (1977) The neuronal basis of a sensory analyzer, the acridid movement detector system. IV. The preference for small field stimuli. J Exp Biol 68:157-185.

Salinas E, Abbott L (1997) Invariant visual responses from attentional gain fields. J Neurophysiol 77:3267-3272.

Sanchez-Vives MV, Nowak LG, McCormick DA (2000a) Cellular mechanisms of long-lasting adaptation in visual cortical neurons in vitro. J Neurosci 20:4286-4299.

Sanchez-Vives MV, Nowak LG, McCormick DA (2000b) Membrane mechanisms underlying contrast adaptation in cat area 17 in vivo. J Neurosci 20:4267-4285.

Sary G, Vogels R, Orban GA (1993) Cue-invariant shape selectivity of macaque inferior temporal neurons. Science 260:995-997.

Schlotterer GR (1977) Response of the locust descending movement detector neuron to rapidly approaching and withdrawing stimuli. Can J Zool 55:1372-1376.

Schwartz EL, Desimone R, Albright TD, Gross CG (1983) Shape recognition and inferior temporal neurons. Proc Natl Acad Sci USA 80:5776-5778. 
Simmons P (1980) Connexions between a movement-detecting visual interneurone and flight motoneurones of a locust. J Exp Biol 86:87-97.

Strausfeld NJ, Nässel DR (1981) Neuroarchitecture of brain regions that subserve the compound eyes of crustacea and insects. In: Comparative physiology and evolution of vision in invertebrates Vol VII, part 6B (Autrum H, ed), pp 1-132. Berlin: Springer.

Sun H, Frost BJF (1998) Computation of different optical variables of looming objects in pigeon nucleus rotundus neurons. Nat Neurosci $1: 296-303$.
Vickery VR (1987) The northern Neartic Orthoptera: their origins and survival. In: Evolutionary biology of Orthopteran insects (Baccetti B, ed), pp 581-591. Chichester, England: Ellis Horwood Ltd.

Whitington PM, Bacon JP (1999) The organization and development of the arthropod ventral nerve chord: insights into arthropod relationships. In: Arthropod relationships (Fortey RA, Thomas RH, eds), pp 349-367. London: Chapman \& Hall.

Zanker JM, Srinivasan MV, Egelhaaf M (1999) Speed tuning in elementary motion detectors of the correlation type. Biol Cybern 80:109116. 